\title{
DISTRIBUCIÓN GEOGRÁFICA Y AFINIDAD POR EL SUSTRATO DE LAS ALGAS VERDES (CHLOROPHYCEAE) BÉNTICAS DE LAS COSTAS MEXICANAS DEL GOlFO de MÉXICO Y MAR CARIBE
}

\author{
Gloria Garduño-Solórzano', José luis Godínez-Ortega², Martha M. Ortega² \\ 'Herbario IZTA, Facultad de Estudios Superiores Iztacala, Universidad Nacional Autónoma de México. Tel 5623-1378. \\ ${ }^{2}$ Instituto de Biología, Universidad Nacional Autónoma de México. Apartado Postal 70-233, México 04510, D.F. \\ Correo-e: ggs@servidor.unam.mx
}

\begin{abstract}
Resumen: Se analizó la distribución geográfica de las algas verdes bénticas de las costas mexicanas del Golfo de México y el Mar Caribe, utilizando un banco de datos que incluye 98 publicaciones taxonómicas del periodo 1846 - 2000, con base en el índice de Jaccard. Se registraron 169 especies de 49 géneros. La mayor riqueza específica corresponde a Cladophora (26 especies) y Caulerpa (16). Se corroboró $70.8 \%$ del total de la ficoflora a través de 69 especies recolectadas por los autores en 25 localidades (40.8\%), y $30 \%$ por medio de la consulta de los herbarios MEXU, ENCB y DUKE. En el esquema ficológico del ambiente marino se registraron 17 hábitats en cinco tipos de costa; en ellos se desarrollan las algas en sustratos duros (62.5\%), suaves (48.4\%), animales (36.8\%), plantas (30\%), flotantes (11\%) y artificiales (12.2\%). A lo largo de las costas de la vertiente atlántica de México, se confirmó la distinción entre dos provincias: una correspondiente a la ficoflora tropical de las costas de Quintana Roo hasta Veracruz (provincia Caribeña) y otra flora de aguas subtropicales, pobre en especies (provincia Caroliniana) donde Cabo Rojo es la frontera que separa la costa tropical y subtropical de México.
\end{abstract}

Palabras clave: algas verdes, Chlorophyceae, distribución geográfica, Golfo de México, Mar Caribe.

\begin{abstract}
The geographical distribution of the green benthic seaweeds from the Mexican coasts of the Gulf of Mexico and the Caribbean Sea was analyzed by means of a data bank that includes 98 taxonomic publications that appeared in the 1846 - 2000 period, based on Jaccard's index. In total, 169 species with 49 genera were recorded, with the highest species richness corresponding to Cladophora (26 species) and Caulerpa (16). Over 70.8\% of all the phycoflora was confirmed based on 69 species collected by the authors in 25 localities (40.8\%), and 30\% through consultation of the MEXU, ENCB and DUKE herbaria. The phycological classification scheme of marine environments included 17 habitats in five coastal types where algae develop on different substrates: hard (62.5\%), soft (48.4\%), animal (36.8\%), plant (30\%), floating (11\%), and artificial (12.2\%). The distinction between two provinces along the Mexican coasts of the Atlantic Ocean Basin was confirmed: one corresponding to a rich tropical phycoflora along the southern coast, from Quintana Roo to Veracruz (Caribbean Province), and the other extending along the subtropical waters and being poor in species (Carolinean Province). Cabo Rojo is the boundary between the tropical and the subtropical coasts of Mexico.
\end{abstract}

Key words: Caribbean Sea, Chlorophyceae, geographic distribution, green seaweeds, Gulf of Mexico.

$\mathbf{E}^{1}$ análisis de la distribución geográfica de las algas verdes bénticas de las costas mexicanas del Golfo de México y del Mar Caribe no ha recibido suficiente atención. Sobre este tema, en México sólo se ha publicado un análisis biogeográfico para el orden Laminariales para las costas de la península de Baja California (Aguilar-Rosas et al., 1990); un estudio sobre la variación biogeográfica de Digenea simplex (Dreckmann y Sentíes, 1994) y la dis- tribución de las macroalgas pardas en la península de Yucatán (Díaz-Martín y Espinoza-Ávalos, 2000). Ortega et al. (2001) señalan que el número de estudios taxonómicos de las Chlorophyceae en este litoral por entidad federativa es, en orden decreciente: Veracruz (41), Quintana Roo (23), Yucatán (21), Tamaulipas (14), Campeche (6) y Tabasco (0).

En las costas de la vertiente atlántica de México la cubierta de algas marinas es tropical con algunos elemen- 
tos subtropicales en el norte. En las playas de Tamaulipas la vegetación algal es pobre e incluso está ausente en algunas áreas, y a medida que se avanza hacia el sur y sureste, la vegetación puede ser exuberante, particularmente en el Mar Caribe (Taylor, 1960). Las investigaciones taxonómicas realizadas hasta ahora en estas costas reúnen principalmente información del piso intermareal donde se desarrollan la mayoría de las especies (Ortega et al., 2001). Sin embargo, pocas publicaciones abordan el estudio de la ficoflora de profundidades entre 4 y $30 \mathrm{~m}$ del infralitoral (Lehman y Tunnel, 1992; Mendoza-González et al., 2000).

La naturaleza física del sustrato influye en el establecimiento, la distribución y la composición de la flora marina. Según el esquema modificado de Sheppard (1995), en el ambiente marino se presentan seis tipos de sustratos: duros (rocas y guijarros), suaves (arena y limo), manglares y estuarios, pastos marinos, arrecifes y pelágicos. El medio marino registrado para las algas bénticas de la información analizada está circunscrito al tipo de costa, hábitat y sustrato. Por ejemplo, las playas arenosas no consolidadas por lo general carecen de vegetación, en contraste con los arrecifes, donde está bien desarrollada. Por otra parte, en las lagunas costeras donde la salinidad se modifica con frecuencia, se desarrollan especies eurihalinas como Chaetomorpha linum, Cladophora sericea, C. glomerata, Ulva flexuosa y Rhizoclonium riparium (Servière-Zaragoza et al., 1992). Otro ambiente lo conforman lugares de arena, con oleaje moderado y la presencia de praderas de pastos marinos o ceibadales (Lot-Helgueras, 1971), donde crecen algas de la familia Udoteaceae de manera exuberante.

En este trabajo se analiza la distribución geográfica y la afinidad por el sustrato de las algas verdes bénticas de las costas mexicanas del Golfo de México y el Mar Caribe, con base en la información publicada, exploraciones ficológicas de los autores y consulta de herbarios.

\section{Materiales y métodos}

Se llevó a cabo una recopilación y revisión de artículos que indican registros de algas verdes (Chlorophyceae) para el área de estudio, comprendidos en el periodo 1846 - 2000. Esto permitió conformar un banco de datos con 4,700

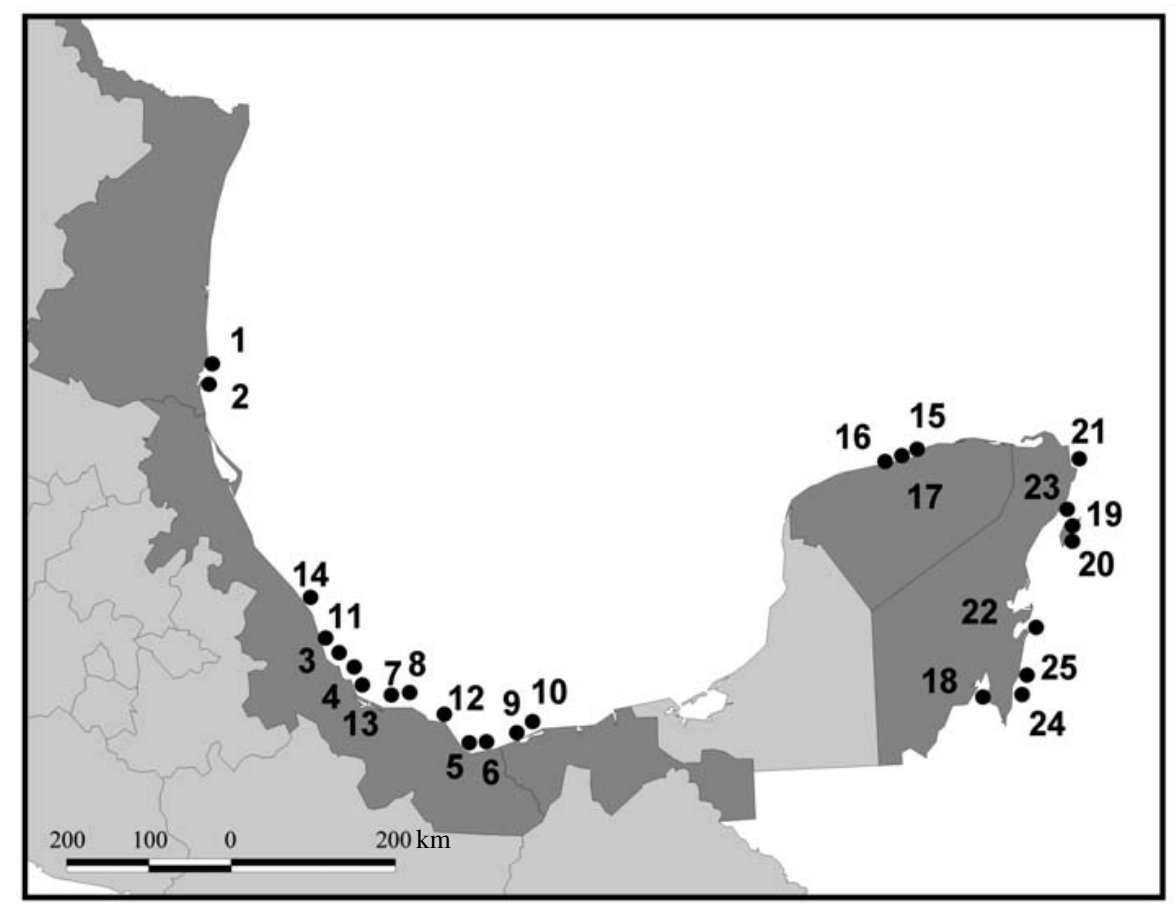

Figura 1. Localidades visitadas en las exploraciones ficológicas con sus coordenadas geográficas. TAMAULIPAS: 1. Escolleras de

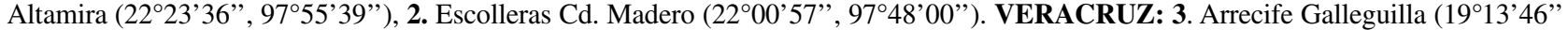
9607’30”), 4. Bajos San Juan de Ulúa (19¹3’19”, 9608’20”), 5. Desembocadura del río Papaloapan (1849’22”, 95³8’06”), 6.

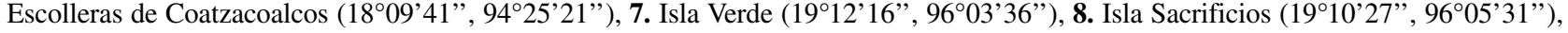

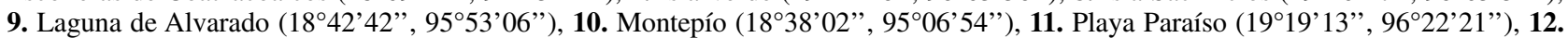

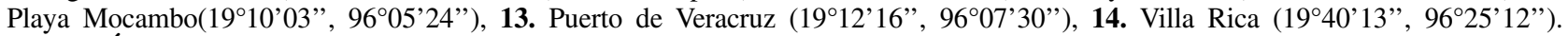

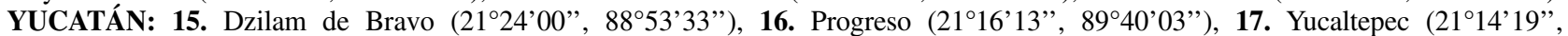

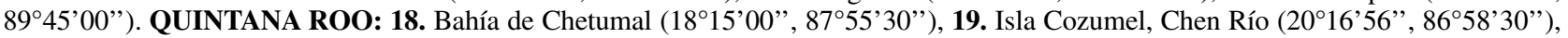
20. Isla Cozumel, Punta Morena ( $\left.20^{\circ} 16^{\prime} 57^{\prime \prime}, 86^{\circ} 58^{\prime} 41^{\prime \prime}\right)$, 21. Isla Mujeres ( $\left.21^{\circ} 23^{\prime} 30^{\prime \prime}, 86^{\circ} 45^{\prime} 00^{\prime \prime}\right)$, 22. Manglar puente Boca Paila

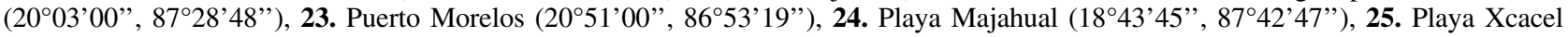
$\left(20^{\circ} 20^{\prime} 27^{\prime \prime}, 87^{\circ} 20^{\prime} 39^{\prime \prime}\right)$. 
registros. Con esta información se elaboró un listado de especies, actualizando la nomenclatura mediante la consulta del Index Nominum Algarum (htpp://128.32.109.44/e-ina. html; Hayden et al., 2003) y excluyendo los registros inciertos. El análisis también reúne datos de campo obtenidos durante las campañas realizadas por los autores, en las que se recolectó material biológico en 25 localidades entre julio de 1991 y febrero de 2001 (figura 1), e incorpora la revisión de colecciones en los herbarios MEXU, ENCB y DUKE (apéndice 1).

Las algas se recolectaron en el piso intermareal directamente con ayuda de una espátula, y en ocasiones con buceo autónomo (SCUBA) en el piso infralitoral, hasta $4 \mathrm{~m}$ de profundidad. El material fue preservado en solución de formaldehído al $4 \%$ en agua de mar. Después de identificarlo, se preservó y depositó en los herbarios IZTA y MEXU. Las especies fueron determinadas siguiendo a Berger y Kaever (1992), Bliding (1963), Littler y Littler (1990, 1991, 1992, 2000) y Taylor (1960).

Distribución geográfica. La distribución geográfica se registró según la presencia o ausencia de cada taxon a lo largo de las costas mexicanas del este del país, tomando como referencia las entidades federativas que abarcan el área de estudio. La similitud ficoflorística se evaluó mediante el coeficiente de asociación de Jaccard:

$$
P=\frac{c}{a+b-c} \times 100
$$

donde $a=$ número de especies de la flora $\mathrm{x}, b=$ número de especies de la flora $\mathrm{y}, c=$ número de especies comunes entre ambas. Se realizó un análisis de clasificación aglomerativa (UPGMA) que usó los valores del índice de Jaccard (van den Hoek, 1975; Rohlf, 1993).

Ambiente. El hábitat fue ordenado según el tipo de sustrato: inorgánico, vegetal, animal, artificial, además de las algas flotantes. A su vez, el sustrato inorgánico se clasificó con base en el tamaño de la partícula. Para su análisis se utilizó la clasificación de costas de Ortiz-Pérez y EspinosaRodríguez (1991) y se estableció una equivalencia con los hábitats registrados en el banco de datos.

Para cada taxon se registró el sustrato inorgánico y de diferentes orígenes donde se desarrolla, por ser éstos algunos de los factores que intervienen en la distribución de las macroalgas bentónicas. Para la clasificación del tamaño de las partículas del sustrato inorgánico se consideró la propuesta de De la Lanza-Espino et al. (1999).

\section{Resultados}

Banco de datos y campañas de recolecta. El análisis de las publicaciones que incluyen el registro de especies de Chlorophyceae permitió registrar 169 taxa específicos, dis- tribuidos en siete órdenes, 18 familias y 49 géneros (apéndice 1). Los géneros con mayor riqueza específica fueron Cladophora (26 especies), Caulerpa (16), Halimeda (11) y Udotea (10), mientras que los géneros monoespecíficos fueron Acicularia, Boodlea, Boodleopsis, Cladocephalus, Cymopolia, Chalmasia, Chamaedoris, Dasycladus, Ernodesmis, Gomontia, Ostreobium, Petrosiphon, Pringsheimiella, Rhipilia, Ulvella, Ulothrix, Valoniopsis y Ventricaria.

Las exploraciones ficológicas realizadas por los autores en 25 localidades localizadas en Tamaulipas, Veracruz, Yucatán y Quintana Roo corroboran la presencia de 69 especies, lo que representa $40.2 \%$ del total de las Chlorophyceae del área de estudio. Casi la tercera parte $(30.2 \%)$ corresponde a material consultado en los herbarios MEXU, ENCB y DUKE. Cabe aclarar que de 29.6\% del total de taxa no se encontró material de herbario de referencia.

En el apéndice 1 se anotan las especies según la clasificación de Wynne (1998), las publicaciones de referencia, la distribución en las cinco entidades federativas registradas, las localidades exploradas y las colecciones de herbario consultadas.

Análisis de clasificación. Con base en el análisis de clasificación de las entidades federativas, en el dendrograma se muestra la relación del grado de similitud entre las entidades federativas (figura 2). Se distinguen básicamente dos grupos, uno constituido por Campeche, Veracruz, Yucatán y Quintana Roo, con similitud hasta de $42 \%$, y otro por Tamaulipas con $26 \%$. El índice de similitud más alto (50\%) se obtuvo para la comparación entre Quintana Roo y Yucatán. También se observó una similitud de estas entidades con Veracruz, pero diferente en relación con Tamaulipas, lo cual permitió inferir la existencia de una frontera biogeográfica entre Tamaulipas y el resto de las entidades federativas adyacentes que divide dos regiones: la subtropical hacia el norte y la tropical hacia el sur.

Ambientes. El cuadro 1 muestra el esquema ficológico general del ambiente marino para las costas del este de México; se señalan 17 hábitats registrados en los cinco tipos de costas determinados por Ortiz-Pérez y EspinosaRodríguez (1991).

Las costa acumulativas son las más comunes; ejemplo de ello son las 37 lagunas costeras, entre las que sobresalen por su extensión la Laguna Madre, Tamiahua, Términos, Celestum y Nichupté. Enseguida las costas biogénicas tienen una alta proporción por encontrarse tres grandes sistemas arrecifales: el Sistema Arrecifal Veracruzano (SAV), constituido por más de 25 islas, arrecifes y bajos enfrente de las costas de Veracruz; el segundo nombrado Banco de Campeche; y finalmente frente a Quintana Roo se localiza 


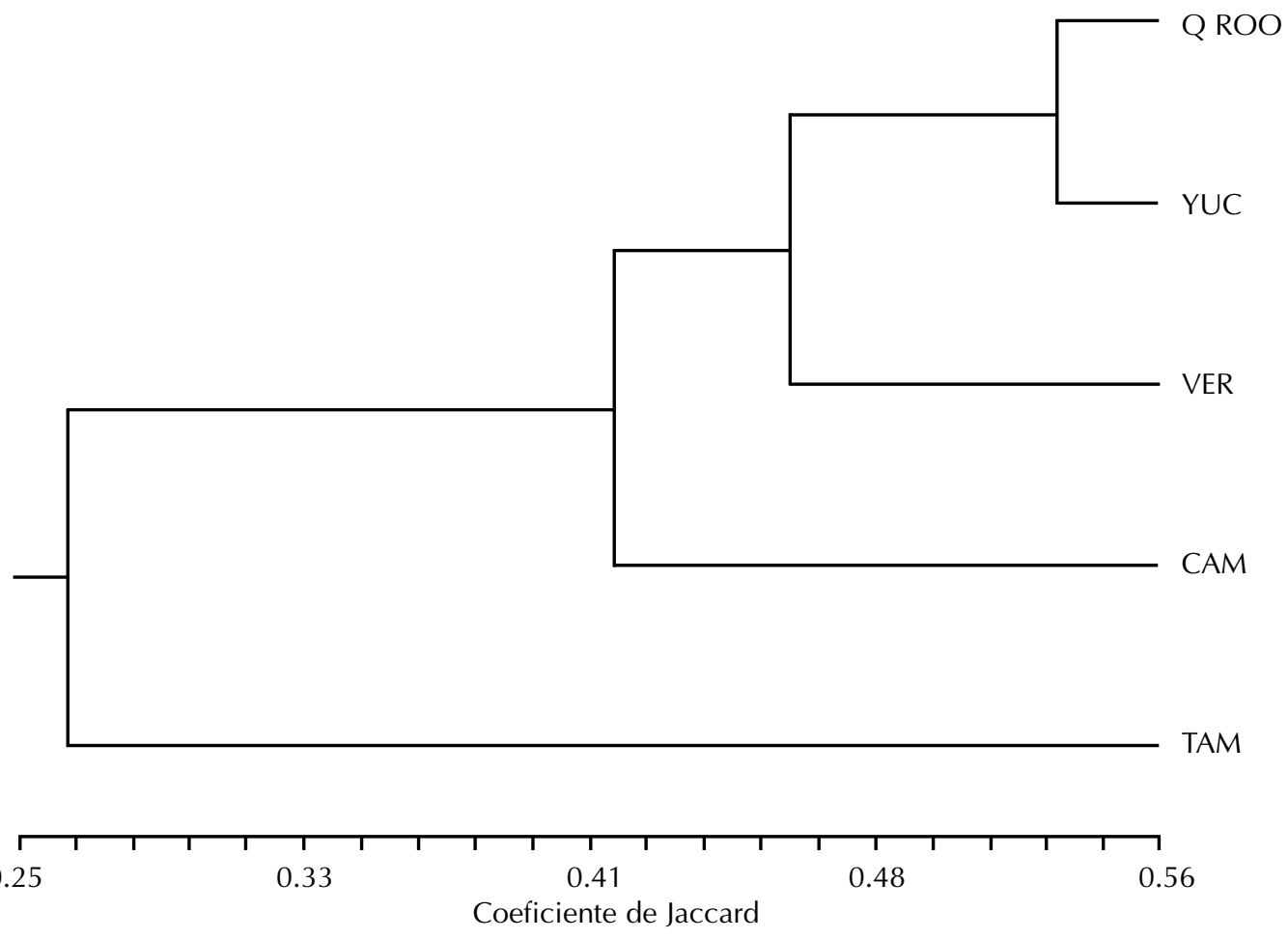

Figura 2. Dendograma de las similitudes florísticas entre las entidades federativas de las costas mexicanas del Golfo de México y Mar Caribe, obtenido con el procedimiento UPGMA.

Cuadro 1. Clasificación de tipos y subtipos de costas en México comparados con los hábitats y sustratos registrados en el área de estudio.

\begin{tabular}{|c|c|c|c|}
\hline \multicolumn{2}{|c|}{ Ortiz-Pérez y Espinosa-Rodríguez (1991) } & \multicolumn{2}{|l|}{ Presente estudio } \\
\hline Tipos de costas & Subtipos de costas & Hábitats & Sustratos \\
\hline 1.-Erosivas (rocosas) & Acantilados (escarpes y roqueríos) & Promontorios rocosos & Duros \\
\hline $\begin{array}{l}\text { 2.-Abrasivo-acumulativas } \\
\text { (mixtas) }\end{array}$ & $\begin{array}{l}\text { Rocosas alternando con playas y/o conos } \\
\text { detríticos y/o abanicos aluviales. } \\
\text { Entrantes con depósitos de playa, alternando } \\
\text { con salientes o puntas rocosas }\end{array}$ & $\begin{array}{l}\text { Afloramiento y puntas } \\
\text { rocosas, morros } \\
\text { Bahías, caletas }\end{array}$ & Duros \\
\hline $\begin{array}{l}\text { 3.-Acumulativas (de } \\
\text { playas bajas arenosas) }\end{array}$ & $\begin{array}{l}\text { Campos de dunas } \\
\text { Cordones litorales } \\
\text { Islas de barrera, barras y flechas litorales } \\
\text { en bocas, lagunas y esteros }\end{array}$ & $\begin{array}{l}\text { Playas arenosas } \\
\text { Barras } \\
\text { Bocas o desembocaduras } \\
\text { Islas de barrera }\end{array}$ & Suaves \\
\hline $\begin{array}{l}\text { 4.-Acumulativas } \\
\text { (potamogénicas y } \\
\text { marismas) }\end{array}$ & $\begin{array}{l}\text { Estuarios y deltas } \\
\text { Llanuras de inundación, manglar y/o } \\
\text { pantano marino }\end{array}$ & $\begin{array}{l}\text { Playas limosas de estuarios } \\
\text { Manglares } \\
\text { Lagunas costeras } \\
\text { Canales de corriente y manglar } \\
\text { Pastos marinos }\end{array}$ & Plantas \\
\hline $\begin{array}{l}\text { 5.-Biogénicas (barrera } \\
\text { coralina) }\end{array}$ & $\begin{array}{l}\text { Corales emergentes } \\
\text { Barrera coralina }\end{array}$ & $\begin{array}{l}\text { Islas coralinas } \\
\text { Arrecifes litorales } \\
\text { Bancos de ostiones }\end{array}$ & $\begin{array}{l}\text { Animales } \\
\text { Flotantes y } \\
\text { artificiales }\end{array}$ \\
\hline
\end{tabular}


el arrecife de barrera más grande del país. El Golfo de México es una provincia distributiva, ya que en él descargan el sistema fluvial Grijalva-Usumacinta junto con el Papaloapan, lo que permite que a lo largo de estas costas se presente una gran diversidad de ambientes desde manglares hasta playas limosas y arenosas. Por último, las costas rocosas y abrasivo acumulativas (mixtas) son las menos comunes en el área de estudio (Contreras-Espinosa, 1993; Toledo-Ocampo, 1996).

Sustratos. Se encontró que el mayor porcentaje de las especies de la flora (60\%) se desarrolla en más de un sustrato, por ejemplo Caulerpa cupressoides, C. racemosa, Cladophora vagabunda y Ulva lactuca crecen en rocas, guijarros, arena y limo, mientras que $26.4 \%$ crece exclusivamente en uno, por ejemplo, Valoniopsis pachynema (rocas) y Udotea spinulosa, U. verticillosa (arena). Este patrón también fue corroborado con los datos obtenidos en las campañas de recolección. Del conjunto total de registros, $13.6 \%$ carecen de información de hábitat, lo que indica que la proporción de la bibliografía que no señala el sustrato es mínima.

En la figura 3 se presenta la cuantificación de cada uno de los tipos de sustratos ocupados por los 169 taxa, tomando en consideración que un organismo puede desarrollarse en más de un sustrato. El porcentaje más alto se observó en los sustratos duros (rocas y guijarros) con $62.5 \%$, seguido por los sustratos suaves y en menor proporción por los relacionados con animales y plantas, los sustratos artificiales y las flotantes. En la figura 4 se muestran los porcentajes de Chlorophyceae, especificando los diferentes sustratos; los porcentajes más altos fueron de origen inorgánicos naturales como rocas $(53.9 \%)$, arena (47.2\%) y guijarros $(32.5 \%)$, los cuales estuvieron seguidos por conchas $(26.9 \%)$, corales $(15.3 \%)$, mangle $(14.1 \%)$ y algas (13.5\%). En contraste, los sustratos menos citados fueron los correspondientes a esponjas $(1.8 \%)$, hidroides $(1.2 \%)$, poliquetos $(1.2 \%)$ y cirripedios $(0.61 \%)$.

\section{Discusión}

Riqueza ficoflorística. Con 169 taxa infragenéricos para el área de estudio, la riqueza ficoflorística es alta, especialmente si se compara con la ficoflora del océano Pacífico mexicano, cuya extensión es mayor, ya que Pedroche et al. (2003) registraron en dicho litoral 173 taxa de algas verdes. Por su parte, Suárez (1973) señala para las costas de Cuba 116 taxa, cifra que también es menor que la registrada en este estudio.

Es necesario verificar la presencia de Cladophora albi$d a, C$. glomerata var. crassior y C. montagneana en aguas tropicales marinas o salobres, ya que algunos autores las ubican para regiones frías y templadas, e incluso en otros casos para aguas dulces (van den Hoek, 1963). Debido a su parecido morfológico, algunos géneros pueden ser confundidos, por ejemplo Blidingia con Enteromorpha, por lo que

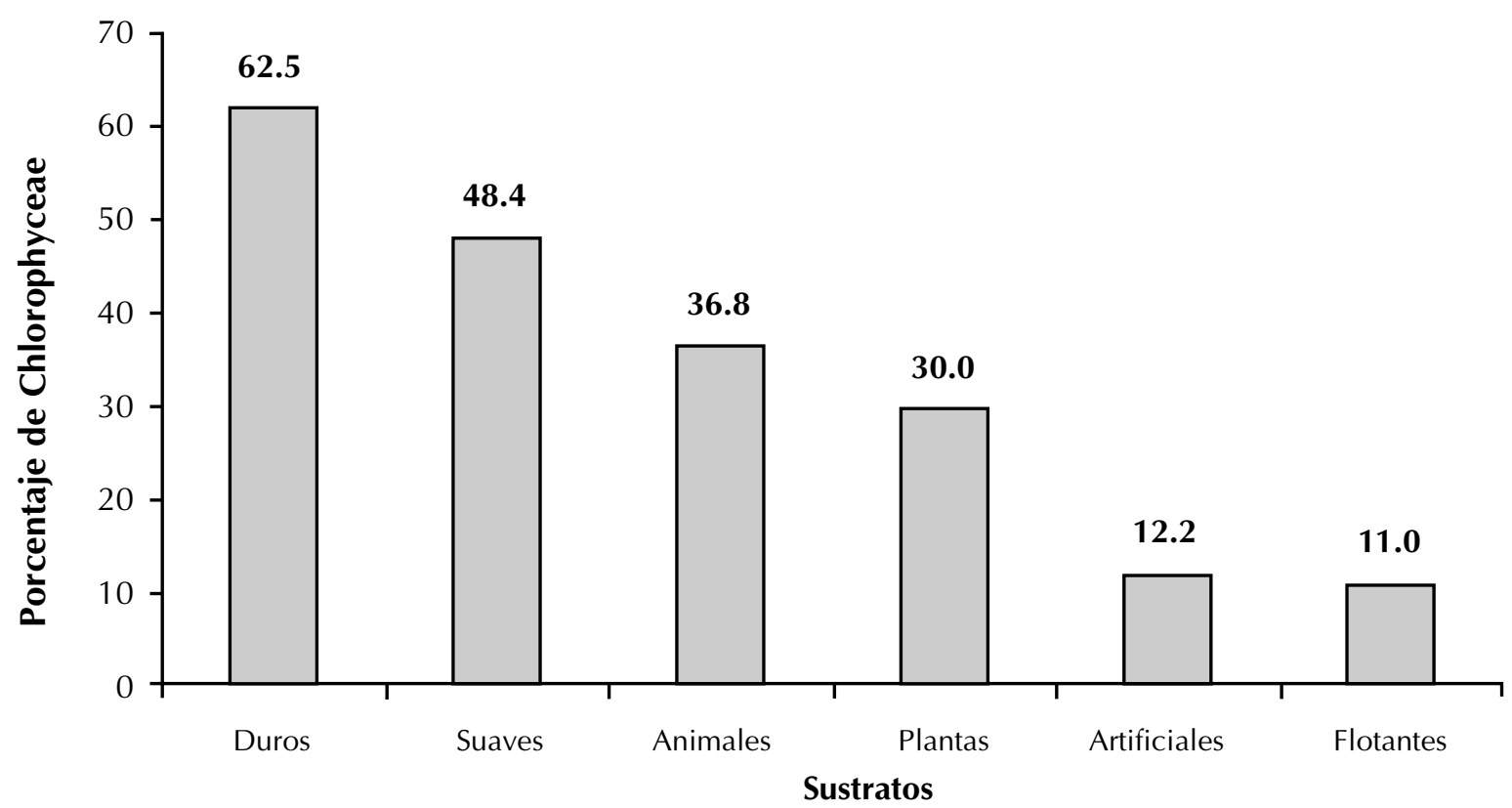

Figura 3. Panorama general de los sustratos ocupados por las Chlorophyceae de las costas mexicanas del Golfo de México y Mar Caribe. 


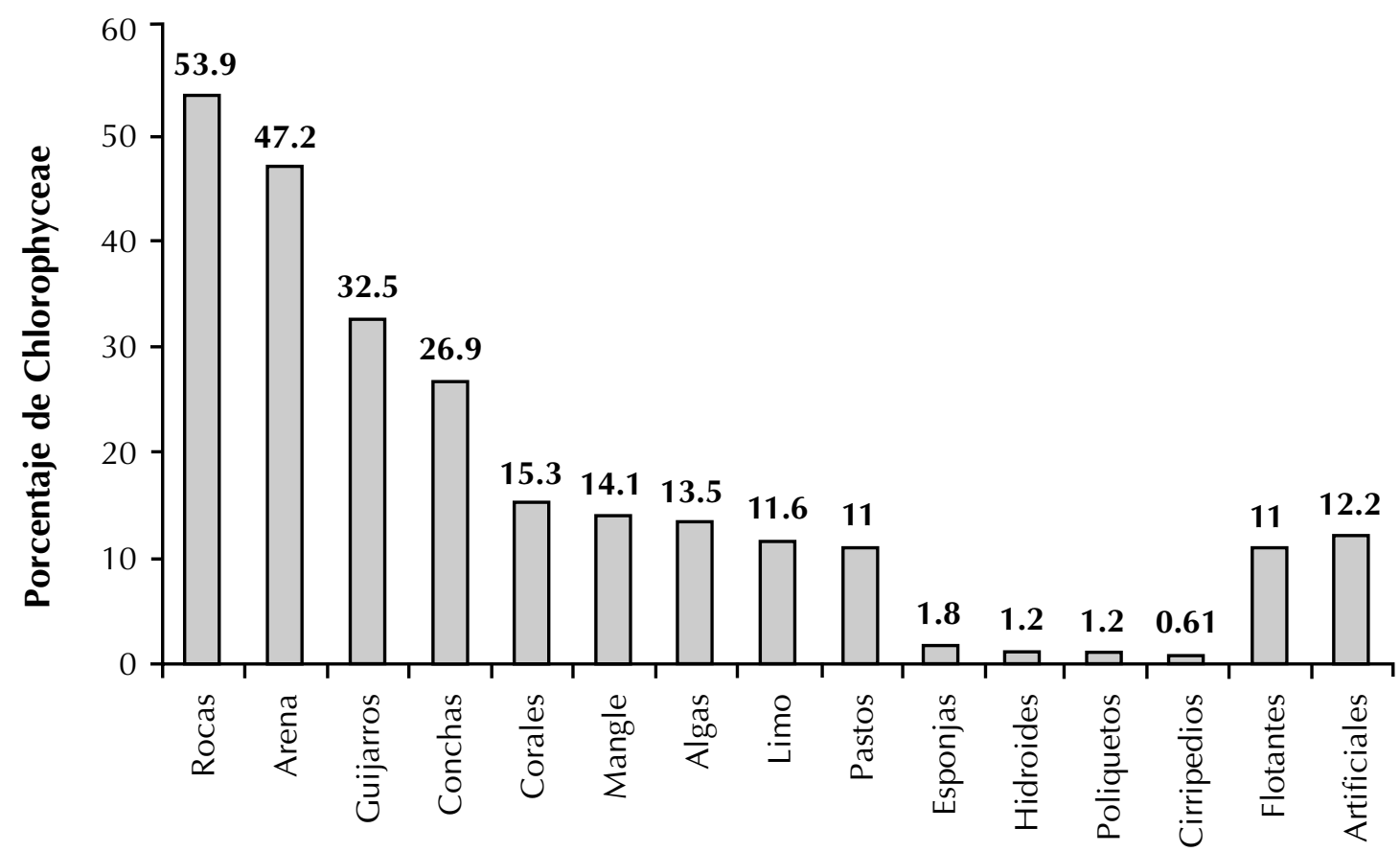

Sustratos

Figura 4. Porcentajes del número de especies de Chlorophyceae sobre los diferentes sustratos.

los registros del primero deben nuevamente confirmarse.

Las colecciones biológicas son herramientas valiosas que pueden apoyar futuros estudios florísticos, monográficos y de revisión, detectar localidades y grupos taxonómicos poco investigados, y que además permiten revalorar algunos taxa con aplicación incierta o errónea, o incluso excluir registros del área de estudio, cuya distribución ha sido citada para regiones geográficas muy distantes y en condiciones ambientales diferentes. Como ejemplo podemos citar a Codium tomentosum, una especie restringida en el Océano Atlántico europeo y el norte de África.

Con respecto al $29.2 \%$ de registros taxonómicos sin colección de referencia (véase apéndice 1), 30 taxa sólo se han citado una vez en este estudio o son especies poco comunes para la costa atlántica de México, o se trata de aquellas que se desarrollan en el piso infralitoral, por ejemplo Blidingia marginata, Caulerpa webbiana, Cladophora albida, Chalmasia antillana, Halimeda lacrimosa, Microdictyon boergesenii, Valoniopsis pachynema, entre otras (Littler y Littler, 2000; Ortega et al., 2001).

Distribución geográfica. El panorama general de la distribución muestra un gradiente latitudinal de norte a sur. Por ejemplo, Cladophora ruchingeri se cita sólo de Tamaulipas. Además, se observa que la mayoría de la flora presenta una distribución continua representada por Ulva fasciata, $U$. lactuca, $U$. flexuosa, $U$. prolifera y Rhizoclonium riparium, entre otras. Sin embargo, también se registran algunas discontinuidades, por ejemplo, las mostradas por Bryopsis hypnoides y B. pennata, las cuales pueden deberse a la variedad del sustrato, la temperatura, la salinidad u otros factores que regulan el desarrollo de la flora marina, así como a las barreras geográficas, el establecimiento de costas artificiales y a la poca exploración en algunas entidades federativas como Campeche y Tabasco. Finalmente, la flora de mayor riqueza específica corresponde a la región caribeña de Quintana Roo, representada por Caulerpa lanuginosa, Chamaedoris peniculum, Valonia aegagropila y V. macrophysa, entre otras.

Los resultados del análisis de clasificación de las entidades federativas se pueden explicar en el hecho de que Quintana Roo y Yucatán se localizan geográficamente en regiones tropicales con formaciones de origen coralino, donde la temperatura, los hábitats y los sustratos favorecen el desarrollo de un gran número de especies de Chlorophyceae. En el Caribe son muy abundantes las Udoteaceae que se implantan en la arena, formando grandes praderas entre los pastos marinos (Thalassia testudinum, Syringodium y Halodule). Sobre las hojas de pastos crece una gran cantidad de epífitas, enriqueciendo la diversidad de estos ambientes (Huerta, 1978). Por su parte, 
las costas de la parte central de Veracruz cuentan con numerosos arrecifes coralinos (Sistema Arrecifal Veracruzano), islas y bajos, además de manglares y costas rocosas, entre otros hábitats, que dan en conjunto un litoral con una alta riqueza algal. Campeche y Tamaulipas cuentan con litorales costeros donde se localizan lagunas costeras (Laguna de Términos y Laguna Madre, respectivamente), donde la salinidad oscila entre 0.20 y $75 \%$, y por lo que sólo crecen organismos eurihalinos. Por su parte, la temperatura del agua desciende hasta $19^{\circ} \mathrm{C}$ en invierno en las costas de Tamaulipas y por lo tanto estos son sitios donde crecen especies subtropicales (ContrerasEspinosa, 1993).

Históricamente, la distribución de las algas marinas en el mundo se ha explicado a través de algunos esquemas biogeográficos. Con base en la temperatura promedio mensual del agua en verano, Setchell (1920) reconoció cinco regiones: boreal superior y boreal austral $\left(-2\right.$ a $\left.10^{\circ} \mathrm{C}\right)$, boreal baja y austral baja $\left(10\right.$ a $\left.15^{\circ} \mathrm{C}\right)$, templado norte y templado sur $\left(15\right.$ a $\left.20^{\circ} \mathrm{C}\right)$, subtropical norte y sur $\left(20\right.$ a $\left.25^{\circ} \mathrm{C}\right)$ y tropical $\left(25\right.$ a $\left.30^{\circ} \mathrm{C}\right)$. Por su parte, Lüning (1990) dividió el medio marino en siete regiones: ártica, templada fría del hemisferio norte, templada cálida del hemisferio norte, tropical, templada cálida del hemisferio sur, templada fría del hemisferio sur, y antártica, con base en un esquema zoogeográfico de Briggs (1974). Para México, Briggs (1974) toma como referencia los estudios de corales e indica el extremo norte de Veracruz (Cabo Rojo, 21 30') como la frontera que divide dos provincias: la subtropical o Provincia Caroliniana y la tropical o Provincia Caribeña. Al respecto, Earle (1972) también señala que la flora algal del Golfo de México tiene afinidades fitogeográficas muy similares con los corales. Al comparar el esquema de distribución del presente estudio con los mencionados anteriormente, se confirma que las costas mexicanas comprenden dos regiones divididas por una frontera situada entre Tamaulipas y Veracruz: hacia el norte la subtropical y hacia el sur la región tropical. La distribución de las algas del área de estudio registró tres floras. El mayor porcentaje de especies de algas verdes se presentó en la región tropical $(80.1 \%)$ y en mínima proporción exclusivamente en la región subtropical $(0.61 \%)$, aunque también se observó una flora común o ubicua que incluye ambas regiones (19.4\%).

Algunos taxa representativos de la región tropical fueron Acetabularia spp., Boodlea composita, Caulerpa spp., Cladophora catenata, C. crispula, C. conferta, C. jongiorum, C. pellucidoidea, Halimeda spp. y Siphonocladus tropicus, los cuales se citan en la literatura como propios de regiones tropicales (Hillis, 1959; van den Hoek, 1982; Lüning, 1990; Berger y Kaever, 1992).

La flora subtropical de Tamaulipas está ejemplificada únicamente por Cladophora ruchingeri, especie citada por van den Hoek (1982) dentro del grupo cálido templado para la costa del Atlántico occidental. La flora común pre- sente en ambas regiones, representada por la familia Ulvaceae, particularmente el género Ulva, presenta una distribución continua desde Tamaulipas hasta Quintana Roo, por lo que el análisis de la información del presente trabajo apoya lo expuesto por Lüning (1990).

Sustratos. Más de la mitad (53.9\%) de las especies han sido recolectadas en sustrato rocoso. Taylor (1960) señala que este sustrato expuesto al oleaje es el lugar donde se localiza una gran diversidad de algas marinas. La textura, el grado de dureza y el color de las rocas influyen sobre las comunidades algales. Por ejemplo, Valonia sp. y Dictyosphaeria spp. pueden albergarse entre los intersticios de las rocas y con ello protegerse del oleaje; en tal caso las estructuras de adhesión tienen modificaciones relacionadas con la superficie utilizada. En contraste, Gomontia polyrhiza y Ostreobium quekettii se desarrollan por la relación química del sustrato exclusivamente dentro de rocas de carbonato de calcio, debido a los iones calcio que pueden tomar (Santelices, 1977).

De las estribaciones del Eje Neovolcánico se desprenden abundantes rocas basálticas ásperas cuyo color negro favorece la absorción de calor y puede provocar el desarrollo de algunas especies de Ulva, Codium y Cladophora. En otros casos, las rocas calcáreas de color blanco con numerosas oquedades, comunes en Quintana Roo y Yucatán, permiten reflejar la luz en beneficio de las algas que las habitan. Acetabularia crenulata, A. polyphysoides, Acicularia schenkii y Cladophora catenata son ejemplos de macroalgas que crecen sobre este tipo de sustratos.

Sobre guijarros o cantos rodados se desarrolla $32.5 \%$ de las especies. Este sustrato está constituido por pequeñas piedras redondas (4 a $64 \mathrm{~mm}$ ) producidas por el desgaste de rocas de mayor tamaño y cuyas rugosidades permiten la adhesión de las macroalgas. Particularmente en localidades insulares las algas se encuentran principalmente del lado de sotavento, es decir, en zonas someras y protegidas del oleaje. En dichas localidades se encontraron representantes de las Dasycladales como Acetabularia farlowii, Acicularia schenckii, Cymopolia barbata, Neomeris annulata, $N$. dumetosa y Chalmasia antillana. Según Santelices (1977), los guijarros de localidades litorales protegidas del oleaje pueden ser colonizados por algas de rápido crecimiento como Blidingia marginata, B. minima, Bryopsis hypnoides, B. pennata, B. plumosa, Cladophora corallicola, C. intextexta, C. liebetruthrii, Cladophoropsis membranaceae, Codium intertextum, C. isthmocladum, C. taylorii, Cymopolia barbata, Dasycladus vermicularis, Ernodesmis verticillata, Siphonocladus rigidus, Ulva compressa, U. flexuosa, U. linza, U. prolifera, Valonia macrophysa y Ventricaria ventricosa. También para lugares expuestos al oleaje, Berger y Kaever (1992) citan a Cymopolia barbata sobre guijarros con arena.

Sobre arena se desarrolla $47.2 \%$ de las especies. Taylor 


\section{Gloria Garduño-Solórzano, José Luis Godínez-Ortega, Martha M. Ortega}

(1954) señala que en estas partículas de $<0.62 \mathrm{~mm}$ de diámetro por lo general se forman desiertos marinos, especialmente en la arena suelta cuya estructura no permite el desarrollo apropiado de algas. Sin embargo, algunas algas verdes presentan mecanismos de adaptación efectivos que les permiten ocupar este hábitat y evitar ser arrancadas por la acción de los agentes físicos como el oleaje. Algunas especies de Chaetomorpha se fijan al sustrato a través de un disco basal lobulado, Rhizoclonium puede tener proyecciones delgadas que constituyen fuertes rizoides, $\mathrm{y}$ Penicillus capitatus, $P$. dumetosus, $P$. lamourouxii y Udotea cyathiformis presentan un sistema rizoidal bien desarrollado que penetra el sustrato (Santelices, 1977). En este sustrato crecen representantes de las familias Caulerpaceae (Caulerpa), Halimedaceae (Halimeda), Cladophoraceae (Cladophora, Chaetomorpha y Rhizoclonium), Udoteaceae (Avrainvillea, Penicillus, Rhipilia, Rhipocephalus, Udotea, Boodleopsis y Cladocephalus) y Dasycladaceae (Batophora, Cymopolia, Dasycladus y Neomeris). La combinación de arena y limo entre los rizomas de pastos marinos (Thalassia, Halodule y Ruppia) ofrece otro sustrato para Cladophoropsis macromeres y Ulva flexuosa (Ortega, 1995). Huerta (1978) y Santelices (1977) mencionan que el desarrollo de pastos marinos o ceibadales desempeña una función importante en la estabilización del sustrato arenoso y limoso en donde las algas pueden desarrollarse adecuadamente, utilizando los espacios que dejan libres los pastos marinos.

Sobre limo se desarrolla $11.6 \%$ de las especies. Este sustrato con partículas de 0.004 a $0.06 \mathrm{~mm}$ de diámetro frecuentemente se acompaña con arena; el crecimiento de praderas de Thalassia testudinum es común y se localiza en los alrededores de las desembocaduras fluviales, estuarios y lagunas costeras. Algunas algas sobre este sustrato fueron Caulerpa peltata, C. mexicana, Chaetomorpha aerea, $C$. linum, Cladophora coelothrix, C. laetevirens, C. vagabunda, Cladophoropsis macromeres, Enteromorpha lingulata, Penicillus lamourouxii, P. pyriformis, Udotea flabellum y Ulva lactuca.

Animales: Sobre animales sésiles (corales, esponjas, hidroides, poliquetos y cirripedios) y animales móviles (moluscos) se desarrollan $36.8 \%$ de las Chlorophyceae. El mayor porcentaje de sustratos animales correspondió a conchas de moluscos (26.9\%), con representantes de las familias Dasycladaceae, Polyphysaceae, Ulvaceae y Anadyomenaceae. Huerta et al. (1987) indicaron que Acetabularia crenulata, Neomeris sp. y Dasycladus vermicularis crecen sobre conchas de los animales móviles como los moluscos. Particularmente en los corales (vivos o muertos) se presentó $15.3 \%$ de las especies. Entre las más comunes se encuentran Avrainvillea nigricans, Anadyomene stellata, Cymopolia barbata, Halimeda opuntia, Neomeris annulata, Petrosiphon adherens y
Ventricaria ventricosa. Más de 30 arrecifes se desarrollan en un intervalo de 2 a $40 \mathrm{~m}$ de profundidad, sobre la plataforma continental atlántica (Toledo-Ocampo, 1996), por lo que no sería difícil que el número de especies de algas sobre animales aumentara en futuras investigaciones dirigidas al conocimiento de las macroalgas en arrecifes. En la zona frontal de los arrecifes de barrera, las comunidades de gorgonáceos y algas son los elementos más conspicuos del sistema (Jordán, 1993). Pocas especies fueron registradas sobre esponjas $(1.8 \%)$, hidroides $(1.2 \%)$, poliquetos $(1.2 \%)$ y cirripedios $(0.61 \%)$, lo cual refleja la falta de estudios en México sobre estas relaciones biológicas.

Vegetales: Las algas que crecen sobre pastos marinos representaron $11 \%$; en su mayoría corresponden a filamentos como Cladophora montagneanea, C. sericea, C. vagabunda y Rhizoclonium riparium. Ibarra-Obando y Ríos (1993) señalan que la distribución de algas sobre los pastos marinos no es homogénea; en general, sólo las hojas maduras constituyen un sustrato fuertemente colonizado.

Las raíces de Rhizophora mangle son excelentes soportes para las algas epirrizas, cuyo porcentaje fue $14.1 \%$, cifra que es ligeramente mayor que las correspondientes a los grupos que crecen sobre otras algas $(13.5 \%)$ y pastos marinos (11\%). Particularmente, Collado-Vides et al. (1995) describen para Caulerpa verticillata estructuras que les permiten a esta especie adherirse a la raíz del mangle con un crecimiento estolonífero.

Sobre otras algas se registró $13.5 \%$ de las especies. Algunos ejemplos de esta relación son Cladophora brassiliana, Chaetomorpha minima, Entocladia viridis, Pedobesia lamourouxii, Phaeophila dendroides, Pringsheimiella scutata, Pseudendoclonium marinum y Ulvella lens.

Algas flotantes. En la superficie de las lagunas costeras flotan masas de filamentos; a este grupo perteneció $11 \%$ de los taxa, por ejemplo Cladophora sericea, Chaetomorpha aerea, C. brachygona, C. gracilis, C. linum, Rhizoclonium africanum, $R$. riparium y Ulothrix flacca (Collado-Vides et al., 1994). Earle (1972) señala que las poblaciones macroscópicas de algas que flotan deben considerarse como flora béntica debido a que originalmente estuvieron fijas a un sustrato. Sin embargo, en algunos casos el material debe tomarse con reserva, ya que puede tratarse de organismos muertos.

Sustratos artificiales. La modificación del ambiente por influencia humana es cada vez más fuerte; las construcciones como escolleras y muelles son un ejemplo de esta transformación. Su estudio es reciente, ya que este tipo de sustrato comenzó a citarse en México a partir de los trabajos de Huerta (1958) y Huerta y Garza-Barrientos (1964), entre otros. Algunos ejemplos de Chlorophyceae que crecen sobre escolleras y muelles son Chaetomorpha antenni- 
na, Cladophora patentiramea, Cladophoropsis membranacea y Ulva fasciata; sobre madera Ulva flexuosa y $U$. compressa; sobre cuerdas de lancha, Rhizoclonium riparium y Chaetomorpha linum; sobre unicel, Derbesia marina; y sobre cable de polipropileno, Ulothrix flacca y Cladophora laetevirens. Las especies que crecen sobre sustratos artificiales representaron $12.2 \%$ del total, lo cual indica que algunas algas han tenido la capacidad de adaptarse a estos materiales.

Futuras investigaciones deberán abordar estudios para Caulerpa para resolver si las condiciones ambientales inducen expresiones morfológicas diferentes, por lo que las observaciones del campo y el crecimiento en cultivo permitirán tomar decisiones taxonómicas (Coppejans, 1992).

\section{Agradecimientos}

A las autoridades del Instituto de Biología y de la FES Iztacala (Universidad Nacional Autónoma de México), por los subsidios otorgados para la realización de numerosas exploraciones ficológicas. A la estación de Puerto Morelos, Quintana Roo, del Instituto de Ciencias del Mar y Limnología (UNAM) por las facilidades brindadas durante la estancia en sus instalaciones y recolecta de material biológico. A la Secretaría de Marina, SEMARNAP y PROFEPA por los permisos y apoyo en las exploraciones ficológicas.

\section{Literatura citada}

Agardh J.G. 1887. Till algernes systematik. Nya bidrag. (Femte afdelningen) Acta Universitatis Lundensis 23:1-174.

Aguilar-Rosas L.E., Aguilar-Rosas M.A., Gómez Pedroso-Cedillo A. y Fernández-Prieto J.A. 1992. Adiciones a la flora marina del Caribe mexicano. Acta Botanica Mexicana 19:77-84.

Aguilar-Rosas M.A., Aguilar-Rosas L.E. y Fernández-Prieto J.A. 1989. Algas marinas bentónicas de la bahía de la Ascensión, Quintana Roo, México. Boletín del Instituto Oceanográfico (Cumaná) 28:67-75.

Aguilar-Rosas M.A. 1990. Algas marinas bentónicas de la Reserva de la Biosfera de Sian Ka'an, Quintana Roo, México. En: Navarro D. y Robinson J.G. Eds. Diversidad Biológica en la Reserva de la Biosfera de Sian Ka'an, Quintana Roo, México, pp. 13-34, Centro de Investigaciones de Quintana Roo y University of Florida, Chetumal.

Aguilar-Rosas R., Aguilar-Rosas L.E. y Ramos-Jardón N.A. 1990. Análisis biogeográfico del orden Laminariales (Phaeophyta) en las costas de la Península de Baja California, México. Investigaciones Marinas CICIMAR 5:107-122.

Arellano-Guillermo A. y Serrano-Islas M.A. 1993. Reserva de Dzilam, Yucatán. En: Salazar-Vallejo S.I. y González N.E. Eds. Biodiversidad Marina y Costera de México, pp. 630-640, Comisión Nacional para el Conocimiento y Uso de la Biodiversidad y Centro de Investigaciones de Quintana Roo, México, D.F.

Barton E.S. 1901. The Genus Halimeda. Siboga-Expeditie Monographe LX, Leiden.
Berger S. y Kaever M.J. 1992. Dasycladales: An Illustrated Monograph of a Fascinating Algal Order. Georg Thieme Verlag, Stuttgart.

Bliding C. 1963. A critical survey of European taxa in Ulvales. Part I. Capsosiphon, Percusaria, Blidingia, Enteromorpha. Opera Botanica 8:1-160.

Briggs J.C. 1974. Marine Zoogeography. McGraw-Hill, Nueva York.

Collado-Vides L. y González-González J. 1993. Macroalgas del sistema lagunar de Nichupté, Quintana Roo. En: SalazarVallejo S.I. y González N.E. Eds. Biodiversidad Marina y Costera de México, pp. 752-760, Comisión Nacional para el Conocimiento y Uso de la Biodiversidad y Centro de Investigaciones de Quintana Roo, México, D.F.

Collado-Vides L., González-González J. y Gold-Morgan M. 1994. A descriptive approach to the floating masses of algae of a Mexican Caribbean coastal lagoon. Botanica Marina 37:391-396.

Collado-Vides L., González-González J. y Ezcurra E. 1995. Patrones de distribución ficoflorística en el sistema lagunar de Nichupté, Quintana Roo, México. Acta Botanica Mexicana 31:19-32.

Contreras-Espinosa F. 1993. Ecosistemas Costeros Mexicanos. Comisión Nacional para el Conocimiento y Uso de la Biodiversidad y Universidad Autónoma Metropolitana, México, D.F.

Coppejans E. 1992. Marine algae of Papua New Guinea (Madang Prov.) 2. A revised and completed list of Caulerpa (Chlorophyta-Caulerpales). Blumea 36:383-410.

Chávez E.A. 1973. Observaciones generales sobre las comunidades del arrecife de Lobos, Veracruz. Anales de la Escuela Nacional de Ciencias Biológicas 20:13-21.

Chávez E.A., Hidalgo E. y Sevilla M.L. 1970. Datos acerca de las comunidades bentónicas del arrecife de Lobos, Veracruz. Revista de la Sociedad Mexicana de Historia Natural 31:211280.

De la Campa de Guzmán S. 1965. Notas preliminares sobre un reconocimiento de la flora marina del estado de Veracruz. Anales del Instituto Nacional de Investigaciones BiológicasPesqueras 1:9-49.

De la Lanza-Espino G., Cáceres-Martínez C., Adame-Martínez S. y Hernández-Pulido S. 1999. Diccionario de Hidrología y Ciencias Afines. Instituto de Biología, Universidad Nacional Autónoma de México y Plaza y Valdés, México, D.F.

Díaz-Martín M.A. y Espinoza-Ávalos J. 2000. Distribution of brown seaweeds (Phaeophyta) in the Yucatan Peninsula, Mexico. Bulletin of Marine Science 66:279-289.

Dreckmann K.M. y Sentíes A. 1994. El alga Digenea simplex (Ceramiales: Rhodomelaceae) en México: variación biogeográfica. Revista de Biología Tropical 42:443-453.

Dreckmann K.M. y Pérez-Hernández M.A. 1994. Macroalgas bentónicas de la laguna de Tampamachoco, Veracruz, México. Revista de Biología Tropical 42:715-717.

Dreckmann K.M., Stout I. y Sentíes-Granados A. 1996. Lista actualizada de las algas marinas bentónicas de Puerto Morelos, Quintana Roo, Caribe mexicano. Polibotanica 3:1-17.

Earle S.A. 1972. Benthic algae and seagrass species in the Gulf of Mexico. En: Bushnell V.C. Ed. Serial Atlas of the Marine Environment, pp. 25-29, American Geographical Society, Nueva York. 


\section{Gloria Garduño-Solórzano, José Luis Godínez-Ortega, Martha M. Ortega}

Flores-Davis J.G. 1993. Clorofíceas del Litoral rocoso de La Mancha, Veracruz. Secretaría de Educación y Cultura, Dirección General de Educación Media Superior y Superior, Xalapa.

Garza-Barrientos M.A. 1977. Primeras consideraciones referentes sobre flora marina del sureste de la República Mexicana. En: Memorias del Simposio Latinoamericano de Oceanografía Biológica (Cumaná, Venezuela, 1975) 2:7-25.

Garza-Barrientos M.A., Martínez-Lozano S. y Escalante M.A. 1984. Contribución al conocimiento de las algas marinas bentónicas de Ciudad Madero, Tamaulipas, México. Phycologia Latinoamericana 2:103-125.

González-Fierro A., Vázquez-Botello A., Villanueva-Fragoso S. y Ponce-Vélez G. 1994. Presencia de metales en sedimentos recientes y organismos de la laguna Sontecomapan, Veracruz, México. Hidrobiológica (México) 4:35-43.

Harvey W.H. 1858. Nereis boreali-americana: or, Contributions to the history of the marine algae of North America. Part III.Chlorospermeae. Smithsonian Contribution Knowledge 10:140.

Hayden H.S., Blomster J., Maggs C.A., Silva P.C., Stanhope M.J. y Waaland R. 2003. Linnaeus was right all along: Ulva and Enteromorpha are not distinct genera. European Journal of Phycology 38:277-294.

Hildebrand H.H. 1957. Estudios biológicos preliminares sobre la Laguna Madre de Tamaulipas. Ciencia (México) 17:151-173.

Hillis L.W. 1959. A revision of the genus Halimeda (order Siphonales). Publications of the Institute of Marine Science 6:321-403.

Huerta L. 1958. Contribución al conocimiento de las algas de los bajos de la Sonda de Campeche, Cozumel e Isla Mujeres. Anales de la Escuela Nacional de Ciencias Biológicas 9:115123.

Huerta L. 1960a. Lista preliminar de las algas marinas del litoral del Estado de Veracruz. Boletín de la Sociedad Botánica de México 25:39-45.

Huerta L. 1960b. Guía de la excursión: Veracruz. [Lista de algas marinas]. En: Primer Congreso Mexicano de Botánica (24 a 26 octubre de 1960), pp. 25-26. Sociedad Botánica de México, México, D.F.

Huerta L. 1961. Flora marina de los alrededores de la Isla Pérez, Arrecife Alacranes, Sonda de Campeche, México. Anales de la Escuela Nacional de Ciencias Biológicas 10:11-22.

Huerta L. 1978. Vegetación acuática y subacuática. En: Rzedowski J. Vegetación de México, pp. 327-339, Limusa, México, D.F.

Huerta L. y Garza-Barrientos M.A. 1964. Algas marinas de la Barra de Tuxpan y de los arrecifes Blanquilla y Lobos. Anales de la Escuela Nacional de Ciencias Biológicas 13:5-21.

Huerta L. y Garza-Barrientos M.A. 1966. Algas marinas del litoral del estado de Campeche. Ciencia (México) 24:193-200.

Huerta L. y Chávez M.L. 1966 [1968]. Presencia de vitamina B12 en algunas algas marinas de las costas de México. Anales de la Escuela Nacional de Ciencias Biológicas 15:9-22.

Huerta L. y Garza-Barrientos M.A. 1980. Contribución al conocimiento de la flora marina de la zona sur del litoral de Quintana Roo, México. Anales de la Escuela Nacional de Ciencias Biológicas 23:25-44.

Huerta L., Chávez M.L.y Sánchez-Rodríguez M.E. 1977. Algas marinas de la Isla de Enmedio, Veracruz. Memorias del
Congreso Nacional de Oceanografía (Guaymas, Sonora, México, 1974) 5:314-325.

Huerta L., Mendoza-González A.C. y Mateo-Cid L.E. 1987. Avance sobre un estudio de las algas marinas de la Península de Yucatán. Phytologia 62:23-53.

Humm H.J. y Hamm D. 1976. New records and range extensions of benthic algae in the Gulf of Mexico. Florida Science 39:4245.

Humm H.J. y Hildebrand H.H. 1962. Marine algae from the Gulf Coast of Texas and Mexico. Publications of the Institute of Marine Science 8:227-268.

Ibarra-Obando S.E. y Ríos R. 1993. Ecosistemas de fanerógamas marinas. En: Salazar Vallejo S.I. y González N.E. Eds. Biodiversidad Marina y Costera de México, pp. 54-65, Comisión Nacional para el Conocimiento y Uso de la Biodiversidad y Centro de Investigaciones de Quintana Roo, México, D.F.

Jordán E., Angot M. y de la Torre R. 1978. Prospección biológica de la laguna de Nichupté, Cancún, Q. R., México: Nota científica. Anales del Centro de Ciencias del Mar y Limnología, Universidad Nacional Autónoma de México 5:179-188.

Jordán E.D. 1993. El ecosistema arrefical coralino del Atlántico mexicano. Revista de la Sociedad Mexicana de Historia Natural Vol. Esp. 44:157-175.

Kim C.S. 1964. Marine algae of Alacrán reef, southern Gulf of Mexico. Tesis Doctoral, Duke University, Durham. 213 pp.

Kornicker L.S., Bonet F., Cann R. y Hoskin C.M. 1959. Alacrán Reef, Campeche Bank, México. Publications of the Institute of Marine Science 6:1-22.

Kützing F.T. 1858. Tabulae Phycologicae. Vol. 8. Nordhausen, Alemania.

Lehman R.L. y Tunnell J.W. Jr. 1992. Species composition and ecology of the macroalgae of Enmedio Reef, Veracruz, Mexico. Texas Journal of Science 44:445-457.

Liebmann F.M. 1846. Beretning om Amerikas Vandplanter af Algernes Classe. Oversigt over det Kongelige Danske Videnskabernes Selskabs Forhandlinger og dets Meldemmers Arbeider 1846:72-77.

Littler D.S. y Littler M.M. 1990. Systematics of Udotea species (Bryopsidales, Chlorophyta) in the tropical western Atlantic. Phycologia 29:206-252.

Littler D.S. y Littler M.M. 1991. Systematics of Anadyomene species (Anadyomenaceae, Chlorophyta) in the tropical western Atlantic. Journal of Phycology 27:101-118.

Littler D.S. y Littler M.M. 1992. Systematics of Avrainvillea (Bryopsidales, Chlorophyta) in the tropical western Atlantic. Phycologia 31:375-418.

Littler D.S. y Littler M.M. 2000. Caribbean Reef Plants. An Identification Guide to the Reef Plants of the Caribbean, Bahamas, Florida and Gulf of Mexico. Offshore Graphics, Washington, D.C.

Lot-Helgueras A. 1971. Estudios sobre fanerógamas marinas en las cercanías de Veracruz, Ver. Anales del Instituto de Biología. Universidad Nacional Autónoma de México. Serie Botánica 42:1-48.

Lüning K. 1990. Seaweeds, their Environment, Biogeography, and Ecophysiology. Wiley, Nueva York.

Martínez-Lozano S. y López-Bautista J.M. 1991. Algas marinas bénticas de Soto la Marina, Tamaulipas, México. Publicaciones Biológicas, Facultad Ciencias Biológicas, 
Universidad Autónoma de Nuevo León (México) 5:13-22.

Martínez-Lozano S. y Villarreal-Rivera L. 1991. Algas marinas de San Fernando, Tamaulipas, México. Publicaciones Biológicas, Facultad Ciencias Biológicas, Universidad Autónoma de Nuevo León (México) 5:9-12.

Martínez-Lozano S. y Guajardo-Ríos O. 1991. Lista sistemática de las algas marinas del Puerto El Mezquital, Matamoros, Tamaulipas, México. Biotam 3:16-26.

Martínez-Lozano S., López-Bautista J.M. y Vázquez-Martínez S. 1992. Flora ficológica marina de Altamira, Tamaulipas. Publicaciones Biológicas, Facultad Ciencias Biológicas, Universidad Autónoma de Nuevo León (México) 6:30-37.

Mateo-Cid L.E. y Mendoza-González A.C. 1991. Algas marinas bénticas de la Isla Cozumel, Quintana Roo, México. Acta Botanica Mexicana 16:57-87.

Mateo-Cid L.E. y Mendoza-González A.C. 1993. Algas marinas poco conocidas de la flora mexicana. X. Derbesia marina (Lyngbye) Solier y D. prolifera W. Taylor (ChlorophytaBryopsidaceae). Anales de la Escuela Nacional de Ciencias Biológicas 38:9-16.

Mateo-Cid L.E., Mendoza-González A.C. y Galicia-García C. 1996. Algas marinas de Isla Verde, Veracruz, México. Acta Botanica Mexicana 36:59-75.

Mendoza-González A.C. y Mateo-Cid L.E. 1985. Contribución al conocimiento de la flora marina bentónica de las Islas Sacrificios y Santiaguillo, Veracruz, México. Phytologia 59:916.

Mendoza-González A.C. y Mateo-Cid L.E. 1992. Algas marinas bentónicas de Isla Mujeres, Quintana Roo, México. Acta Botanica Mexicana 19:37-61.

Mendoza-González A.C., Mateo-Cid L.E. y Searles R.B. 2000. New records of benthic marine algae from Isla Cozumel, Mexico: Phaeophyta and Chlorophyta. Bulletin of Marine Science 66:119-130.

Murray G. 1889a. Catalogue of the marine algae of the West Indian region. Journal of Botany 27:237-242, 257-262, 298305.

Murray G. 1889b. Catalogue of the Marine Algae of the West Indian Region. Dulau, Londres.

Murray G. 1891. On new species of Caulerpa, with observations on the position of the genus. Transactions of the Linnean Society of London, Botany 3:207-213.

Novelo-Retana A. 1978. La vegetación de la Estación Biológica El Morro de la Mancha, Veracruz. Biotica 3:9-21.

Ochoa y Villagómez I. 1887. Vegetación espontánea y repoblación de los médanos de la zona litoral de Veracruz. Informe presentado a la Secretaría de Fomento. Memorias de la Secretaría de Fomento, México (1883-1885), 3:641-655.

Orozco-Vega H. y Dreckmann K.M. 1995. Microalgas [Macroalgas] estuarinas del litoral mexicano del Golfo de México. Cryptogamie Algologie 16:189-198.

Ortega M.M. 1995. Observaciones del fitobentos de la laguna de Términos, Campeche, México. Anales del Instituto de Biología. Universidad Nacional Autónoma de México. Serie Botánica 66:1-36.

Ortega M.M., Godínez J.L. y Garduño-Solórzano G. 2001. Catálogo de Algas Bénticas de las Costas Mexicanas del Golfo de México y Mar Caribe. Instituto de Biología, Universidad Nacional Autónoma de México, México, D.F.

Ortiz-Pérez M.A. y Espinosa-Rodríguez L.M. 1991. Clasificación geomorfológica de las costas de México. Geografía y Desarrollo 2:2-9.

Piña C., Ortega M.M. y Landeros D. 1983. Contribución al estudio de la composición química del alga mexicana Ulva fasciata Delile. Anales del Instituto de Biología, Universidad Nacional Autónoma de México, Serie Botánica 54:243-246.

Pedroche F.F., Silva P.C., Aguilar-Rosas L.E., Dreckann K.M. y Aguilar-Rosas R. 2003. Catálogo de las algas marinas bentónicas del Pacífico Mexicano. I. Chlorophyta. En: Memorias del IV Congreso Mexicano de Ficología. Editado por Sociedad Ficológica de México, A.C. (7-11 abril, 2003. Mérida), p. 75.

Quintana-Molina J.R. 1980. La Zonación Rocosa Intermareal de Playa Paraíso, Veracruz. Universidad Autónoma Metropolitana, México, D.F.

Quintana-Molina J.R. 1991. Resultados del programa de investigaciones en arrecifes veracruzanos del laboratorio de sistemas bentónicos litorales. Hidrobiológica (México) 1:73-86.

Quintana-Molina J.R., Ramos-Cárdenas A., Miranda-Arce M.G. y de Lara-Isassi G. 1981a. Catálogo de las Algas Macroscópicas de la Zona de Intermareas de Playa Paraíso, Veracruz. Universidad Autónoma Metropolitana, México, D.F. Quintana-Molina J.R., Ramos-Cárdenas A., Miranda-Arce M.G. y de Lara Isassi G. 1981b. Contribución al conocimiento de la flora ficológica de Playa Paraíso, Ver., México. En: Memorias del Simposio Latioamericano de Oceanografia Biológica (Acapulco, Guerrero, México, 1981) 7:387-405.

Rigby J.K. y McIntire W.G. 1966. The Isla de Lobos and associated reefs, Veracruz, Mexico. Brigham Young University, Science Bulletin, Geological Series 13:3-46.

Rohlf F.J. 1993. NTSYS-PC (Version 1.8). Numerical Taxonomy and Multivariate Analysis System. Applied Biostatistics, Nueva York.

Sánchez-Rodríguez M.E. 1963. Datos relativos a los manglares de México. Anales de la Escuela Nacional de Ciencias Biológicas 12:61-72.

Sánchez-Rodríguez M.E. 1965 [1967]. Flora marina de Monte Pío, Edo. de Veracruz, México. Anales de la Escuela Nacional de Ciencias Biológicas 14:9-18.

Sánchez-Rodríguez M.E., Flores-Davis G. y Ramírez-Rodríguez A. 1975. Trayecto Playa Paraíso - Villa Rica - Boca Andrea. En: Guías Botánicas de Excursiones en México, pp. 77-82, Sociedad Botánica de México, Xalapa.

Sánchez-Rodríguez M.E. 1980. Ficoflora del sustrato rocoso dentro de las costas del Golfo de México, México. Boletim do Instituto Oceanografico (São Paulo) 29:347-350.

Santelices B. 1977. Ecología de Algas Marinas Bentónicas. Efectos de Factores Ambientales. Universidad Católica de Chile, Santiago.

Servière-Zaragoza E., Collado-Vides L. y González-González J. 1992. Caracterización ficológica de la Laguna de Bojórquez, Quintana Roo, México. Caribbean Journal of Science 28:126133.

Setchell W.A. 1920. The temperature interval in the geographical distribution of marine algae. Science 52:187-190.

Sheppard C.R.C. 1995. Biological communities of tropical oceans. En: Nierenberg W.A. Ed. Encyclopedia of Environmental Biology Vol. 1, Academic Press, San Diego. pp. 277-289.

Silva P.C. 1960. Codium (Chlorophyta) in the tropical western Atlantic. Nova Hedwigia 1:497-536. 


\section{Gloria Garduño-Solórzano, José Luis Godínez-Ortega, Martha M. Ortega}

Silva P.C. 1962. Comparison of algal floristic patterns in the Pacific with those in the Atlantic and Indian oceans, with special reference to Codium. En: Proceedings of the Ninth Pacific Sciences Congress (Bangkok, 1957) 4:201-216.

Suárez A.M. 1973. Catálogo de las Algas Cubanas. Universidad de La Habana, La Habana.

Taylor W.R. 1935. Marine algae from the Yucatan Peninsula. Publications of the Carnegie Institution of Washington 461:115-124.

Taylor W.R. 1941a. Notes on the marine algae of Texas. Papers of the Michigan Academy of Science 26:69-79.

Taylor W.R. 1941b. Tropical marine algae of the Arthur Schott Herbarium. Publications of the Field Museum of Natural History, Botanical Series 20:87-104.

Taylor W.R. 1954. Sketch of the character of the marine algal vegetation of the shores of the Gulf of Mexico. Fishery Bulletin of the Fish and Wildlife Service 55:177-192.

Taylor W.R. 1960. Marine Algae of the Eastern Tropical and Subtropical Coast of the Americas. University of Michigan, Ann Arbor.

Taylor W.R. 1972. Marine algae of the Smithsonian-Bredin expedition to Yucatan-1960. Bulletin of Marine Science 22:34-44.

Toledo-Ocampo A. 1996. Marco conceptual: caracterización ambiental del Golfo de México. En: Botello A.V., Rojas-
Galaviz J.L., Benítez J.G. y Zarate-Lomelí D. Eds. Golfo de México, Contaminación e Impacto Ambiental: Diagnóstico y Tendencias, pp. 1-24, Universidad Autónoma de Campeche, Campeche.

Torruco D., González M.A. y Liddell W.D. 1993. Integración ecológica de grupos funcionales en la laguna arrecifal de Alacranes Yucatán, México. Brenesia 39-40:37-49.

van den Hoek C. 1963. Revision to the European Species of Cladophora. E.J. Brill, Leiden.

van den Hoek C. 1975. Phytogeographic provinces along the coasts of the northern Atlantic Ocean. Phycological Reviews 3, Phycologia 14:317-330.

van den Hoek C. 1982. A Taxonomic Revision of the American Species of Cladophora (Chlorophyceae) in the North Atlantic Ocean and their Geographic Distribution. North-Holland Publishing Company, Amsterdam.

Villalobos A. 1971. Estudios ecológicos en un arrecife coralino en Veracruz, México. En: Symposium on Investigations and Resources of the Caribbean Sea and Adjacent Regions. (Curaçao, 1971), pp. 531-545, CICAR, UNESCO y FAO.

Wynne M.J. 1998. A checklist of benthic marine algae of the tropical and subtropical western Atlantic: first revision. Beihefte zur Nova Hedwigia 116:1-155.

Fecha de recepción: 15 de julio de 2003

Versión corregida: 29 de enero de 2005

Aceptado: 29 de enero de 2005 
Apéndice 1. Matriz de algas verdes bénticas de las costas mexicanas del Golfo de México y Mar Caribe con base en la información bibliográfica, recolectas por los autores y la revisión de los ejemplares de herbario consultados. Los números arábigos del 1 al 25 (en negritas) señalan las localidades exploradas (véase figura 1). Los números subrayados después de las autoridades de cada taxón indican la referencia donde la especie fue citada. La columna de colección indica el número de ejemplar. Asimismo se incluye la matriz para calcular el índice de Jaccard donde $\mathrm{P}=$ presencia y $\mathrm{A}=$ ausencia en las cinco entidades federativas registradas y con ello llevar a cabo el análisis de clasificación por UPGMA.

\begin{tabular}{|c|c|c|c|c|c|c|}
\hline Taxa & Q. ROO & YUC. & CAM. & VER. & TAM. & $\begin{array}{c}\text { Herbario } \\
\text { consultado }\end{array}$ \\
\hline \multicolumn{7}{|l|}{ CHLOROPHYCEAE } \\
\hline \multicolumn{7}{|l|}{ CTENOCLADALES } \\
\hline \multicolumn{7}{|l|}{ ULVELLACEAE } \\
\hline Entocladia ventriculosa (Børgesen) Taylor 54, 61 & A & A & $\mathrm{P}$ & A & A & \\
\hline E. viridis Reinke $39,32,3436,45,54,61$ & $\mathrm{P}$ & $\mathrm{P}$ & A & A & A & DUKE 10769 \\
\hline Pringsheimiella scutata (Reinke) Marchewianka $\underline{36}$ & A & $P$ & A & 12 & A & IZTA 899 \\
\hline $\begin{array}{l}\text { Pseudendoclonium marinum (Reinke) Aleem } \\
\text { et Schulz } \underline{32,36} \\
\text { Ulvella lens P. Crouan et H. Crouan } 15,30,32,34 \text {, }\end{array}$ & $\mathrm{P}$ & $\mathrm{P}$ & A & A & A & \\
\hline$\underline{36,39,45,46,49,70,71}$ & $\mathrm{P}$ & $\mathrm{P}$ & A & 14 & $\mathrm{P}$ & IZTA 1003 \\
\hline \multicolumn{7}{|l|}{ ULOTRICHALES } \\
\hline \multicolumn{7}{|l|}{ ULOTRICHACEAE } \\
\hline Ulothrix flacca (Dillwyn) Thuret $7,8,9,13,45,60,72$ & $\mathrm{P}$ & A & A & 11 & $\mathrm{P}$ & IZTA 209 \\
\hline \multicolumn{7}{|l|}{ PHAEOPHILALES } \\
\hline \multicolumn{7}{|l|}{ PHAEOPHILACEAE } \\
\hline \multicolumn{7}{|l|}{ Phaeophila dendroides (P. Crouan et H. Crouan) } \\
\hline Batters $\underline{32,36,49,53,61}$ & $\mathrm{P}$ & $\mathrm{P}$ & $\mathrm{P}$ & A & A & IZTA 1093 \\
\hline \multicolumn{7}{|l|}{ ULVALES } \\
\hline \multicolumn{7}{|l|}{ GOMONTIACEAE } \\
\hline $\begin{array}{l}\text { Gomontia polyrhiza (Lagerheim) Bornet et } \\
\text { Flahault } \underline{34,36,78}\end{array}$ & $\mathrm{P}$ & $\mathrm{P}$ & A & $\mathrm{P}$ & A & DUKE 09645 \\
\hline \multicolumn{7}{|l|}{ MONOSTROMATACEAE } \\
\hline Blidingia marginata (J. Agardh) P. Dangeard ex Bliding $\underline{26}$ & A & $P$ & A & A & A & \\
\hline B. minima (Nägeli ex Kützing) Kylin $13,27,29,30,32,60$ & $\mathrm{P}$ & $\mathrm{P}$ & $\mathrm{P}$ & $\mathrm{P}$ & A & \\
\hline \multicolumn{7}{|l|}{ ULVACEAE } \\
\hline Enteromorpha bulbosa (Suhr) Montagne 12 & A & A & A & $\mathrm{P}$ & A & \\
\hline E. chaetomorphoides Børgesen $7,8,32, \overline{36}, 53,72$ & $\mathrm{P}$ & $\mathrm{P}$ & $\mathrm{P}$ & A & A & ENCB 6520 \\
\hline $\begin{array}{l}\text { E. clathrata (Roth) Greville } 13,32,34,36,45,46,53,60 \\
\text { E. lingulata J. Agardh } 11,12,15,23,25,26,28,32, \\
\quad 34,36,36,45,46,47,48,58,60,61,63,65,66,\end{array}$ & $\mathrm{P}$ & $P$ & A & $\mathrm{P}$ & $\mathrm{P}$ & DUKE 09321 \\
\hline $70,71,75,76,77,80$ & A & $P$ & $\mathrm{P}$ & $3,11,12,13$ & $\mathrm{P}$ & IZTA 852 \\
\hline 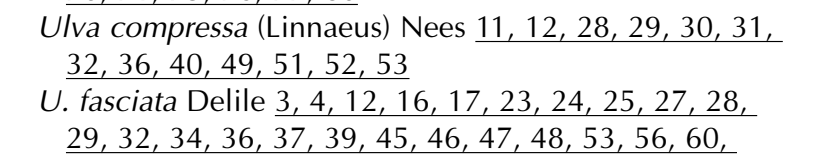 & $P$ & $\mathrm{P}$ & $\mathrm{P}$ & $3,5,7$ & A & IZTA 497 \\
\hline$\underline{61,62,69,70}$ & $\mathrm{P}$ & $P$ & $\mathrm{P}$ & $3,6,9,12,13$ & 2 & IZTA 297 \\
\hline $\begin{array}{l}\text { U. flexuosa (Wulfen) J. Agardh } 3,4,7,8,12,14,16,17 \text {, } \\
24,26,28,29,30,31,32,34,45,46,47,49,60,69,\end{array}$ & & & & & & \\
\hline $70,71,72,76$ & $\mathrm{P}$ & $P$ & $\mathrm{P}$ & 7,11 & $\mathrm{P}$ & IZTA 845 \\
\hline $\begin{array}{l}U . \text { intestinalis (Linnaeus) Nees } 12,60 \\
U . \text { lactuca Linnaeus } 3,4,7,13,14,15,16,26,30,32,36 \text {, }\end{array}$ & A & A & A & 12 & A & IZTA 851 \\
\hline $45,46,47,48,49,52,53,58,60,63,65,66,70,71,72$ & $\mathrm{P}$ & 16 & $\mathrm{P}$ & $3,7,10,11,12$ & $\mathrm{P}$ & IZTA 834 \\
\hline $\begin{array}{l}\text { U. linza (Linnaeus) J. Agardh } \underline{32,53} \\
U . \text { prolifera (O. Müller) J. Agardh } 7,8,26,28,29,31 \text {, }\end{array}$ & $\mathrm{P}$ & $\mathrm{P}$ & A & A & A & ENCB 6513 \\
\hline
\end{tabular}


Apéndice 1. Continuación

\begin{tabular}{|c|c|c|c|c|c|}
\hline Taxa & Q. ROO YUC. & CAM. & VER. & TAM. & $\begin{array}{r}\text { Herbario } \\
\text { consultado }\end{array}$ \\
\hline$\underline{32,34,36,45,70,72}$ & $P$ & $P$ & 3 & $P$ & IZTA 8 \\
\hline U. rigida C. Agardh $\underline{3,12}, 32,34,47,49,65,78$ & A & $P$ & 3,12 & A & IZTA 296 \\
\hline
\end{tabular}

\section{CLADOPHORALES}

\section{ANADYOMENACEAE}

Anadyomene saldanhae Joly et Oliveira $\underline{54}$

A. menziesii (J. Gray) J. Agardh 19, 32, 42, 53, 55, 56

A. stellata (Wulfen) C. Agardh 3, 4, 7, 9, 12, 15, 16, $24,30,32,36,39,40,42,49,51,53,54,69,70$,

$$
71,72,78
$$

Microdictyon boergesenii Setchell 39

M. marinum (Bory de Saint-Vicent) Silva $\underline{54}$

$\begin{array}{cccccc}\text { P } & \text { A } & \text { A } & \text { A } & \text { A } & \\ \text { P } & \text { A } & \text { A } & \mathbf{1 1} & \text { A } & \text { IZTA 905 } \\ & & & & & \\ \mathbf{1 9} & \text { P } & \text { A } & \mathbf{7 , 1 1} & \text { P } & \text { IZTA 570 } \\ \text { A } & \text { A } & \text { A } & \text { P } & \text { A } & \\ \text { P } & \text { A } & \text { A } & \text { A } & \text { A } & \text { ENCB 13086 }\end{array}$

\section{CLADOPHORACEAE}

Chaetomorpha aerea (Dillwyn) Kützing $\underline{3,12,14,17,}$ $32,49,53,61$

C. antennina (Bory) Kützing 14, 15, 16, 17, 23, 25, 29, $32,34,45,49,53,61,69,70,71$

C. brachygona Harvey 12, 19, 24, 28, 30, 32, 34, 49, $\underline{53,55,56}$

C. clavata Kützing 49

C. crassa (C. Agardh) Kützing 32, 39, 49, 53

C. geniculata (Montagne) Montagne 30,32

C. gracilis Kützing 7, 8, 11, 16, 28, 29, 30, 32, 36, 49, 72

C. linum (O. Müller) Kützing 3, 4, 7, 8, 9, 14, 16, 29, 30, 32, 36, 45, 46, 47, 49, 53, 54, 61, 72

C. minima Collis et Hervey 29, 30, 31, 32, 36

Cladophora albida (Nees) Kützing 26, 32, 46, 54, 80

C. brasiliana Martens 14, 16, 30, 32

C. catenata (Linnaeus) Kützing 3, 4, 14, 24, 28, 29, 32, $34,36,49,53,54,78$

C. coelothrix Kützing 29, 32, 36, 51

C. conferta P. Crouan et H. Crouan $\underline{7,9}$

C. constricta Collins $\underline{32,36}$

C. corallicola Børgesen 26, 31, 32

C. crispula Vickers $32,49,53$

C. crystallina (Roth) Kützing 7, 9, 28, 30, 32, 49

C. dalmatica Kützing 13, 45, 49, 60

C. flexuosa (O. Müller) Kützing 12, 28, 34

C. glomerata (Linnaeus) Kützing 28, 49

C. intertexta Collins 26, 32

C. jongiorum van den Hoek 2, 3, 4

C. laetevirens (Dillwyn) Kützing $\underline{26}$

C. lehmanniana (Lindenberg) Kützing $\underline{49}$

C. liebetruthii Grunow 31

C. montagneana Kützing 7, 9, 12, 14, 16, 17, 19, 24, $\underline{28,29,31,32,34,51,60}$

C. patentiramea (Montagne) Kützing 23

C. pellucida (Hudson) Kützing 32

C. pellucidoidea van den Hoek $\underline{7,9}$

C. prolifera (Roth) Kützing 13, 60, 70

C. ruchingeri (C. Agardh) Kützing $\underline{46}$

C. sericea (Hudson) Kützing $7,8, \overline{17}, 34,36,49$, $\underline{60,72,80}$

\begin{tabular}{|c|c|c|c|c|c|}
\hline $\mathrm{P}$ & A & $\mathrm{P}$ & $\mathrm{P}$ & $\mathrm{P}$ & IZTA 950 \\
\hline $\mathrm{P}$ & A & $\mathrm{P}$ & 12 & 2 & IZTA 313 \\
\hline $\mathrm{P}$ & $\mathrm{P}$ & A & $P$ & $\mathrm{P}$ & ENCB 3106 \\
\hline $\mathrm{P}$ & A & A & 11 & A & IZTA 875 \\
\hline 19 & A & $A$ & $P$ & A & IZTA 399 \\
\hline $\mathrm{P}$ & A & A & A & A & \\
\hline $\mathrm{P}$ & $\mathrm{P}$ & $P$ & $\mathrm{P}$ & A & DUKE 08380 \\
\hline $\mathrm{P}$ & $\mathrm{P}$ & $\mathrm{P}$ & A & $\mathrm{P}$ & ENCB 6551 \\
\hline $\mathrm{P}$ & $P$ & $\mathrm{P}$ & $\mathrm{P}$ & $A$ & \\
\hline $\mathrm{P}$ & $P$ & A & $\mathrm{P}$ & $P$ & \\
\hline $\mathrm{P}$ & $\mathrm{P}$ & $\mathrm{P}$ & A & A & \\
\hline $\mathrm{P}$ & $\mathrm{P}$ & $\mathrm{P}$ & $\mathrm{P}$ & A & ENCB 6577 \\
\hline A & $P$ & $P$ & $P$ & A & ENCB 10656 \\
\hline $\mathrm{P}$ & A & A & A & A & \\
\hline A & $\mathrm{P}$ & A & A & A & \\
\hline $\mathrm{P}$ & $P$ & $A$ & $P$ & $A$ & \\
\hline $\mathrm{P}$ & A & A & A & A & \\
\hline $\mathrm{P}$ & A & A & $\mathrm{P}$ & A & ENCB 8515 \\
\hline $\mathrm{P}$ & A & A & $\mathrm{P}$ & $\mathrm{P}$ & \\
\hline A & A & A & $\mathrm{P}$ & $P$ & \\
\hline $\mathrm{P}$ & A & A & $\mathrm{P}$ & $A$ & ENCB 8496 \\
\hline A & $P$ & A & A & A & ENCB 1621 \\
\hline $\mathrm{P}$ & A & A & A & A & \\
\hline A & $P$ & $A$ & A & $P$ & ENCB 22 \\
\hline $\mathrm{P}$ & A & A & A & $A$ & \\
\hline A & A & A & $\mathrm{P}$ & A & \\
\hline $\mathrm{P}$ & $\mathrm{P}$ & $\mathrm{P}$ & $\mathrm{P}$ & $\mathrm{P}$ & ENCB 7909 \\
\hline $\mathrm{P}$ & A & A & A & $A$ & \\
\hline $\mathrm{P}$ & A & A & A & A & \\
\hline $\mathrm{P}$ & A & A & A & A & \\
\hline A & A & A & $\mathrm{P}$ & $A$ & \\
\hline A & A & A & A & $\mathrm{P}$ & \\
\hline $\mathrm{P}$ & $\mathrm{P}$ & $A$ & 11,12 & $\mathrm{P}$ & IZTA 896 \\
\hline
\end{tabular}


Apéndice 1. Continuación

\begin{tabular}{|c|c|c|c|c|c|c|}
\hline Taxa & Q. ROO Y & YUC. & CAM. & VER. & TAM. & $\begin{array}{l}\text { Herbario } \\
\text { consultado }\end{array}$ \\
\hline C. socialis Kützing $\underline{51}$ & A & A & A & 7 & A & IZTA 612 \\
\hline \multicolumn{7}{|l|}{ C. submarina P. Crouan et H. Crouan ex Schramm } \\
\hline $\begin{array}{l}\text { C. vagabunda (Linnaeus) van den Hoek } 3,4,7,9,12,14 \\
\begin{array}{l}15,16,17,21,24,25,26,28,29,32,34,36,45,46, \\
47,48,49,53,54,58,61,70,71,75,77\end{array}\end{array}$ & $\frac{4,}{4}$ & $\mathrm{P}$ & $\mathrm{P}$ & $\mathrm{P}$ & $P$ & IZTA I026 \\
\hline Rhizoclonium africanum Kützing $7,8,9,32,49,61$ & 18,21 & $\mathrm{P}$ & $\mathrm{P}$ & A & A & IZTA 735 \\
\hline R. crassipellitum W. West et G. West $7,9,72$ & $\mathrm{P}$ & A & A & A & A & IZTA 898 \\
\hline R. riparium (Roth) Harvey $\underline{3,34,36, \overline{49,}}$ & $P$ & $P$ & $\mathrm{P}$ & $\mathrm{P}$ & $\mathrm{P}$ & ENCB 8485 \\
\hline R. tortuosum (Dillwyn) Kützing $\underline{8,13,15,49,60,70}$ & $P$ & A & A & $\mathrm{P}$ & A & \\
\hline \multicolumn{7}{|l|}{ SIPHONOCLADACEAE } \\
\hline Boodlea composita (Harvey) Brand 16, 36, 51, 53, 78 & $P$ & $\mathrm{P}$ & A & 4,7 & A & IZTA 610 \\
\hline \multicolumn{7}{|l|}{ Cladophoropsis macromeres Taylor $\underline{3,4,7,8,9,14,16,}$} \\
\hline $32,36,47,49,51,53,54,72,78$ & $P$ & $\mathrm{P}$ & $\mathrm{P}$ & 7,11 & $P$ & IZTA 302 \\
\hline \multicolumn{7}{|l|}{$\begin{array}{l}\text { C. membranacea (Hofman Bang ex C. Agardh) Børgesen } \\
3,4,7,9,11,14,15,16,18,23,28,30,331,32,336 \text {, }\end{array}$} \\
\hline$\underline{49,52,51,53,61,68,70,71,72,77,78}$ & $19,21,25$ & $P$ & $P$ & 11 & A & IZTA 728 \\
\hline \multicolumn{7}{|l|}{$\begin{array}{l}\text { Dictyosphaeria cavernosa (Forsskål) Børgesen } \underline{1,3,4,} \\
11,12,16,23,24,25,26,28,30,31,32,36,37,\end{array}$} \\
\hline D. ocellata (Howe) Olsen-Stojkovich 15, 24, 27 & $\mathrm{P}$ & $\mathrm{P}$ & A & $\mathrm{P}$ & A & ENCB8408 \\
\hline D. versluysii Weber-van Bosse $\underline{78}$ & $P$ & A & A & A & A & \\
\hline Petrosiphon adhaerens Howe $\underline{3,36}$ & $P$ & $\mathrm{P}$ & A & A & A & DUKE 10107 \\
\hline \multicolumn{7}{|l|}{ Phyllodictyon anastomosans (Harvey) Kraft et Wynne } \\
\hline $30,32,51$ & $P$ & A & A & A & A & ENCB 7907 \\
\hline P. pulcherrimum J. Gray 55, 56 & $P$ & A & A & A & A & \\
\hline Siphonocladus rigidus Howe $2,3,4,26,32,36$ & 23 & $\mathrm{P}$ & A & A & A & IZTA 1454 \\
\hline \multicolumn{7}{|l|}{$\begin{array}{l}\text { Ventricaria ventricosa (J. Agardh) Olsen et J. West } \underline{3,4} \text {, } \\
11,12,16,23,24,25,26,28,30,31,32,34,36,\end{array}$} \\
\hline$\underline{37,44,49,52,53,54,64,78,79}$ & 24,25 & $\mathrm{P}$ & A & 7 & A & IZTA 972 \\
\hline \multicolumn{7}{|l|}{ VALONIACEAE } \\
\hline Ernodesmis verticillata (Kützing) Børgesen $\underline{51}$ & 22 & A & $\mathrm{P}$ & A & A & IZTA 322 \\
\hline Valonia aegagropila C. Agardh $\underline{3,53,65}$ & 25 & A & A & A & A & IZTA 794 \\
\hline V. macrophysa Kützing $3,4,16,30,32,49,53,54$ & $\mathrm{P}$ & A & A & A & A & ENCB 8383 \\
\hline V. utricularis (Roth) C. Agardh $3,16,30,32,36,49,54$ & $P$ & $P$ & A & A & A & \\
\hline Valoniopsis pachynema (Martens) Børgesen $\underline{30}$ & $P$ & A & A & A & A & \\
\hline \multicolumn{7}{|l|}{ BRYOPSIDALES } \\
\hline \multicolumn{7}{|l|}{ BRYOPSIDACEAE } \\
\hline $\begin{array}{l}\text { Bryopsis halliae Taylor } 2,3 \\
B \text { hypnoides Lamouroux } 15,31,32,34,45,46\end{array}$ & $P$ & A & A & A & A & \\
\hline$\underline{49,51,52,53}$ & $P$ & $\mathrm{P}$ & A & $\mathrm{P}$ & $P$ & ENCB 7936 \\
\hline $\begin{array}{l}\text { B. pennata Lamouroux } 1,14,15,16,17,30,32 \text {, } \\
36,47,48,49,51,53,54,70,78\end{array}$ & 20 & $P$ & A & 7,14 & 1 & IZTA 567 \\
\hline B. plumosa (Hudson) C. Agardh $14,32,39,45$, & & & & & & \\
\hline $46,47,60,61,75,77$ & 20 & $\mathrm{P}$ & $\mathrm{P}$ & 7 & $\mathrm{P}$ & IZTA 568 \\
\hline B. ramulosa Montagne 15,61 & A & A & $\mathrm{P}$ & $\mathrm{P}$ & A & \\
\hline Derbesia marina (Lyngbye) Solier 32, 49, 50, 52, 54 & $\mathrm{P}$ & $\mathrm{P}$ & A & $\mathrm{P}$ & A & ENCB 10206 \\
\hline D. osterhoutii (L. Blinks et A. Blinks) Page 26,32 & $P$ & $\mathrm{P}$ & A & A & A & ENCB 3630 \\
\hline
\end{tabular}


Apéndice 1. Continuación

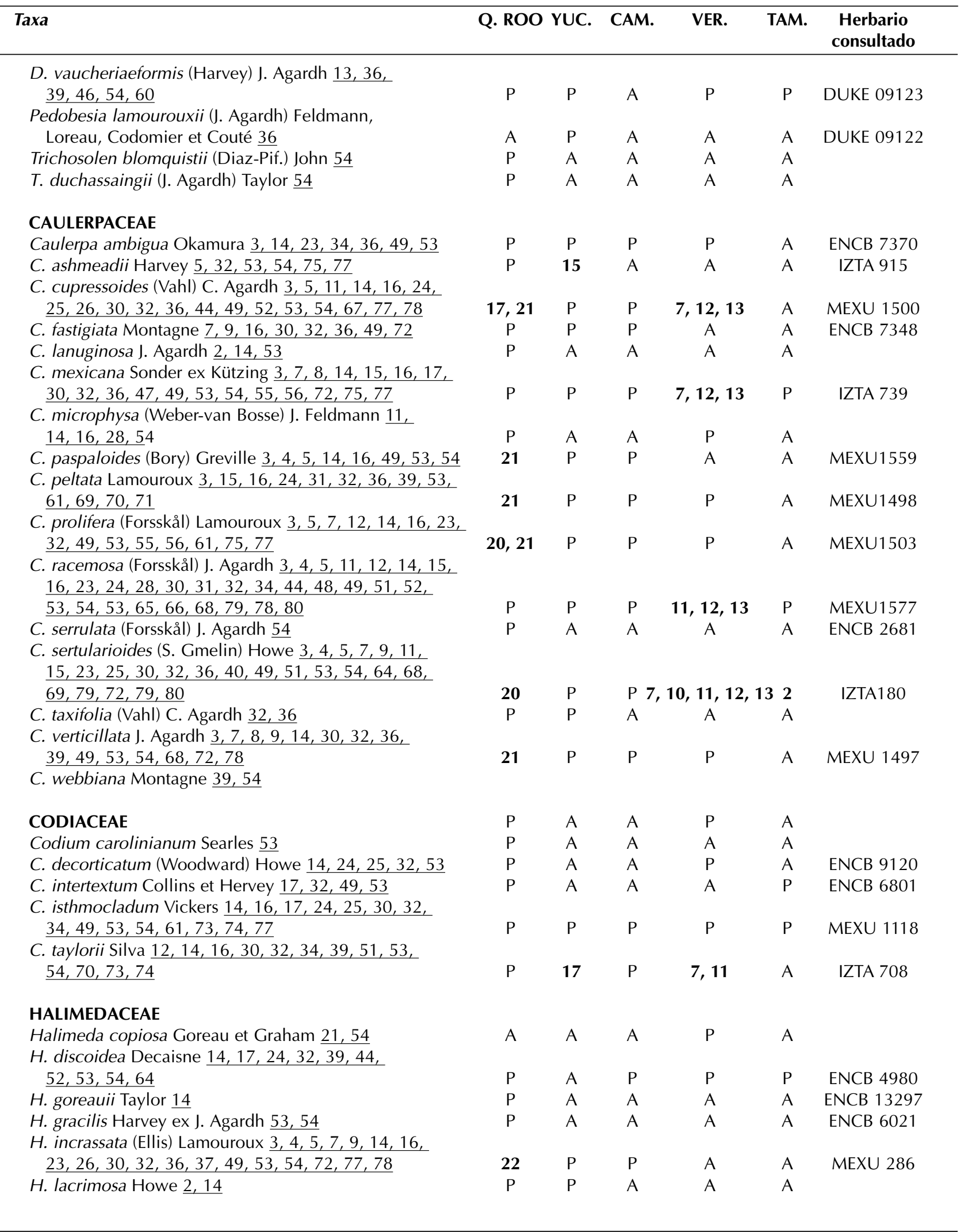


Apéndice 1. Continuación

\begin{tabular}{|c|c|c|c|c|c|c|}
\hline Taxa & Q. ROO & YUC. & CAM. & VER. & TAM. & $\begin{array}{l}\text { Herbario } \\
\text { consultado }\end{array}$ \\
\hline $\begin{array}{l}\text { H. monile (Ellis et Solander) Lamouroux } \underline{3,4,14,} \\
16,26,32,36,49,53,54,68,78\end{array}$ & 20,25 & $P$ & A & A & A & MEXU 285 \\
\hline $\begin{array}{l}\text { H. opuntia (Linnaeus) Lamouroux } 3,4,11,12,14, \\
\begin{array}{l}16,23,24,25,26,28,29,30,31,32,34,36,37, \\
44,49,51,52,53,54,55,56,59,70,71,78,80\end{array}\end{array}$ & 20 & 16 & $P$ & 7 & A & MEX|। 200 \\
\hline H. scabra Howe $3,4,14,15,16,30,32,36,49,53,78$ & 25 & $\mathrm{P}$ & A & 7 & A & IZTA 1018 \\
\hline H. simulans Howe $7,9,14,32,36,49,54$ & $\mathrm{P}$ & $P$ & A & A & A & ENCB 3100 \\
\hline $\begin{array}{l}\text { H. tuna (Ellis et Solander) Lamouroux } \underline{3,4,6,7,9,12,} \\
\qquad \begin{array}{l}14,21,23,24,26,30,31,32,34,36,44,49,51, \\
\underline{53,54,70,75,77,79,80}\end{array}\end{array}$ & $P$ & $P$ & $P$ & 7 & A & IZTA 190 \\
\hline $\begin{array}{l}\text { OSTREOBIACEAE } \\
\text { Ostreobium quekettii Bornet et Flahault } \underline{36}\end{array}$ & A & $P$ & A & 11 & A & IZTA 1118 \\
\hline UDOTEACEAE & & & & & & \\
\hline Avrainvillea asarifolia Børgesen $\underline{3,4,14,32,49}$ & $\mathrm{P}$ & A & A & A & A & ENCB 7488 \\
\hline A. elliottii A. Gepp et E. Gepp $\underline{49,53,54}$ & $P$ & A & A & A & A & ENCB 6053 \\
\hline A. levis Howe $30,32,36,43$ & $P$ & $\mathrm{P}$ & A & A & A & \\
\hline A. longicaulis (Kützing) Murray et Boodle $3,4,7$, & & & & & & \\
\hline $9,14,16,30,32,36,43,49,53,54,68,72,78$ & 24 & $P$ & A & $P$ & A & MEXU 1648 \\
\hline A. mazei Murray et Boodle $\underline{43}$ & $\mathrm{P}$ & A & A & A & A & \\
\hline $\begin{array}{l}\text { A. nigricans Decaisne } 3,4, \overline{5}, 7,9,11,14,16,28 \text {, } \\
30,32,36,49,53,54,72,78\end{array}$ & 24 & $\mathrm{P}$ & A & $P$ & A & MEXU 1644 \\
\hline $\begin{array}{l}\text { A. rawsonii (Dickie) Howe } \underline{3,14}, 16,30,32,49, \\
53,54,78\end{array}$ & 24 & A & A & A & A & IZTA 729 \\
\hline $\begin{array}{l}\text { Boodleopsis pusilla (Collins) Taylor, Joly et Bernatowicz } \\
7,9,54\end{array}$ & 25 & A & A & A & A & IZTA 1455 \\
\hline 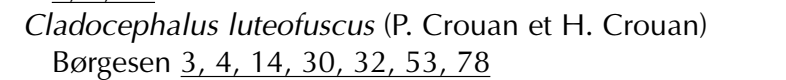 & $P$ & A & A & A & A & ENCB 6022 \\
\hline $\begin{array}{l}\text { Penicillus capitatus Lamarck } 3,4,5,7,9,12,14,16 \text {, } \\
23,26,30,32,35,36,49,53,54,68,72,78\end{array}$ & $17,19,20$ & $P$ & $\mathrm{P}$ & $\mathrm{P}$ & A & IZTA 734 \\
\hline $\begin{array}{l}\text { P. dumetosus (Lamouroux) Blainville } \underline{3,4,14,16,29,} \\
\underline{30,32,49,53,54,78}\end{array}$ & 20 & $P$ & $P$ & A & A & MEXU 1575 \\
\hline $\begin{array}{l}\text { P. lamourouxii Decaisne } 3,4,7,9,14,16,23,30, \\
32,38,49,53,54,72,77,78\end{array}$ & 24 & $\mathrm{P}$ & A & $\mathrm{P}$ & $P$ & MEXU 1563 \\
\hline $\begin{array}{l}\text { P. pyriformis A. Gepp et E. Gepp } 3,14,16,23,26 \text {, } \\
30,32,36,49,53,54,78\end{array}$ & 24 & $P$ & $\mathrm{P}$ & A & A & MEXU 1565 \\
\hline Rhipilia tomentosa Kützing $3,4,14,32,49,53$ & 23 & A & A & A & A & IZTA 387 \\
\hline $\begin{array}{l}\text { Rhipiliopsis profunda (Eiseman et Earle) Norris et Blair } \underline{54} \\
\text { Rhipocephalus oblongus (Decaisne) Kützing }\end{array}$ & $\mathrm{P}$ & A & A & A & A & ENCB 12819 \\
\hline $14,16,32,49,51$ & $P$ & $P$ & A & A & A & \\
\hline $\begin{array}{l}\text { R. phoenix (Ellis et Solander) } 15,36,39,53 \\
\text { Udotea conglutinata (Ellis et Solander) Lamouroux }\end{array}$ & $P$ & $P$ & $\mathrm{P}$ & $\mathrm{P}$ & A & IZTA 1227 \\
\hline $3,4,14,16,30,32,49,53$ & $P$ & $P$ & $\mathrm{P}$ & A & A & ENCB 6126 \\
\hline U. cyathiformis Decaisne $14,32,36,39,49,53,54$ & $\mathrm{P}$ & $P$ & $\mathrm{P}$ & $P$ & A & ENCB 6119 \\
\hline $\begin{array}{l}\text { U. dixonii Littler et Littler } \overline{54} \\
\text { U. flabellum (Ellis et Solander) Howe } 3,4,7,11,14\end{array}$ & 25 & A & A & A & A & IZTA 790 \\
\hline $16,23,29,30,32,36,49,53,54,78$ & 22 & $\mathrm{P}$ & $\mathrm{P}$ & $\mathrm{P}$ & A & IZTA 289 \\
\hline$U$. looensis Littler et Littler $\underline{54}$ & $\mathrm{P}$ & A & A & A & A & ENCB 12749 \\
\hline U. occidentalis A. Gepp et E. Gepp 14, 32, 36, 53, 54 & 25 & $P$ & A & A & A & IZTA 796 \\
\hline U. spinulosa Howe $14,30,32,36, \overline{39,41,49,53,54}$ & $P$ & $\mathrm{P}$ & A & $\mathrm{P}$ & A & ENCB 6128 \\
\hline U. unistratea Littler et Littler $\underline{54}$ & $P$ & A & A & A & A & ENCB 12797 \\
\hline U. verticillosa A. Gepp et E. Gepp 32, 53 & $P$ & A & A & A & A & \\
\hline U. wilsonii A. Gepp, E. Gepp et Howe 14, 32, 53, 54 & $\mathrm{P}$ & A & A & A & A & ENCB 6124 \\
\hline
\end{tabular}


Apéndice 1. Continuación

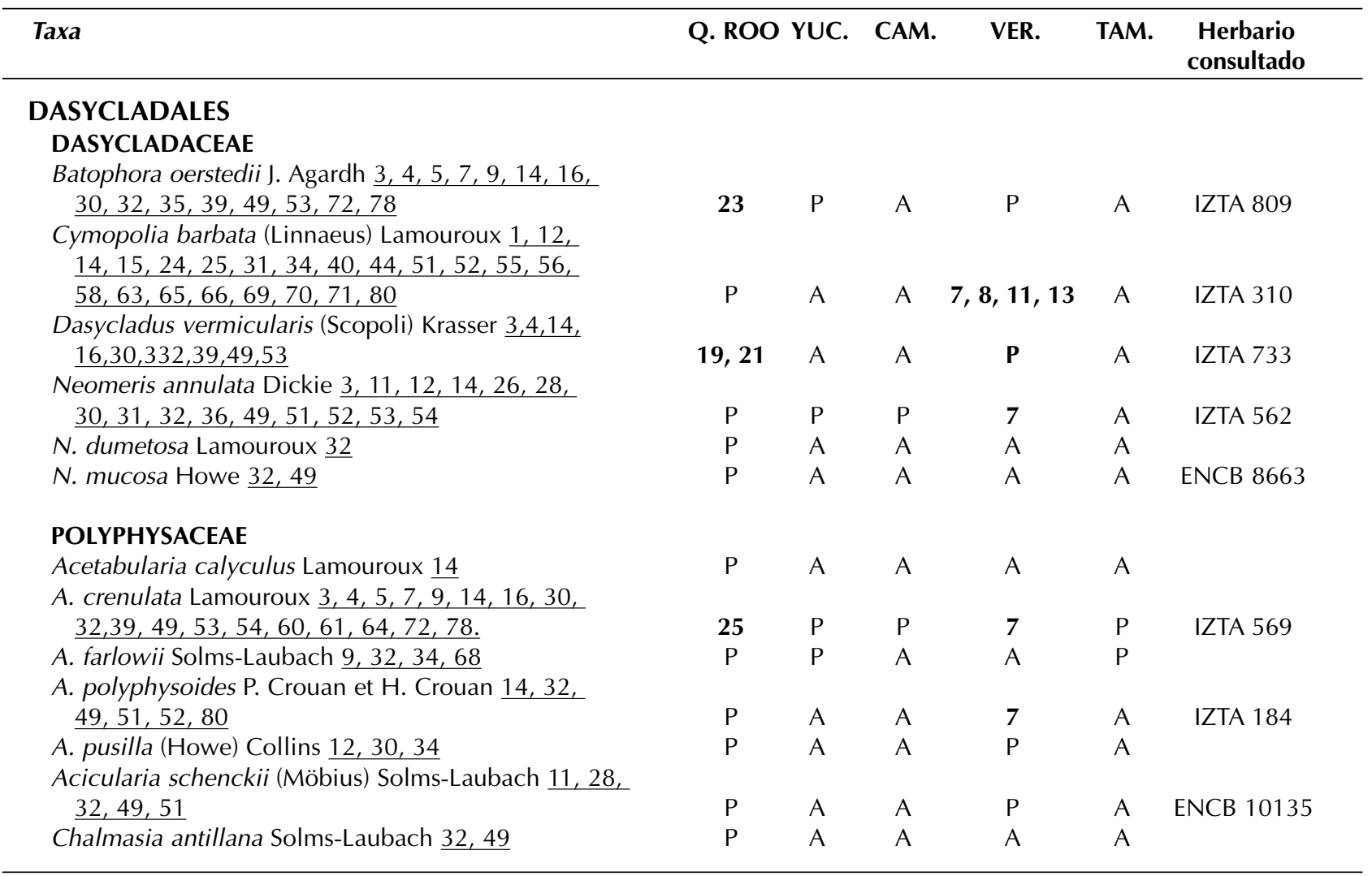

1.- Agardh, 1887. 2.- Aguilar-Rosas et al., 1992. 3.- Aguilar-Rosas, 1990. 4.- Aguilar-Rosas et al., 1989. 5.- ArellanoGuillermo y Serrano-Islas, 1993. 6.- Barton, 1901. 7.- Collado-Vides y González-González, 1993. 8.- Collado-Vides et al.,1994. 9.- Collado-Vides et al., 1995. 10.- Chávez, 1973. 11.- Chávez et al., 1970. 12.- De la Campa, 1965. 13.Dreckmann y Pérez-Hernández, 1994. 14.- Dreckmann et al., 1996. 15.- Flores-Davis, 1993. 16.- Garza-Barrientos, 1977. 17.- Garza-Barrientos et al., 1984. 18.- González-Fierro et al., 1994. 19.- Harvey, 1858. 20.- Hildebrand, 1957. 21.- Hillis, 1959. 22.- van den Hoek, 1982. 23.- Huerta, 1958. 24.- Huerta, 1960a. 25.- Huerta, 1960b. 26.-Huerta, 1961. 27.- Huerta y Chávez, 1966 [1968]. 28.- Huerta y Garza-Barrientos, 1964. 29.- Huerta y Garza-Barrientos, 1966. 30.- Huerta y GarzaBarrientos, 1980. 31.- Huerta et al., 1977. 32.- Huerta et al., 1987. 33.- Humm y Hamm, 1976. 34.- Humm y Hildebrand, 1962. 35.- Jordán et al., 1978. 36.- Kim, 1964. 37.- Kornicker et al.,1959. 38.- Kützing, 1858. 39.- Lehman y Tunnell Jr., 1992. 40.- Liebmann, 1846. 41.- Littler y Littler, 1990. 42.- Littler y Littler, 1991. 43.- Littler y Littler, 1992. 44.- LotHelgueras, 1971. 45.- Martínez-Lozano y López-Bautista, 1991. 46.- Martínez-Lozano y Guajardo-Ríos, 1991. 47.Martínez-Lozano y Villarreal-Rivera, 1991. 48.- Martínez-Lozano et al., 1992. 49.- Mateo-Cid y Mendoza-González, 1991. 50.- Mateo-Cid y Mendoza-González, 1993. 51.- Mateo-Cid et al., 1996. 52.- Mendoza-González y Mateo-Cid, 1985. 53.Mendoza-González y Mateo-Cid, 1992. 54.- Mendoza-González et al., 2000. 55.- Murray, 1889a. 56.- Murray, 1889b. 57.Murray, 1891. 58.- Novelo-Retana, 1978. 59.- Ochoa y Villagómez, 1887. 60.- Orozco-Vega y Dreckmann, 1995. 61.Ortega, 1995. 62.- Piña et al., 1983. 63.- Quintana-Molina, 1980. 64.- Quintana-Molina, 1991. 65.- Quintana-Molina et al., 1981a. 66.- Quintana-Molina et al., 1981b. 67.- Rigby y McIntire, 1966. 68.- Sánchez-Rodríguez, 1963. 69.- SánchezRodríguez, 1965 [1967]. 70.- Sánchez-Rodríguez, 1980. 71.- Sánchez-Rodríguez et al., 1975. 72.- Servière-Zaragoza et al., 1992. 73.- Silva, 1960. 74.- Silva, 1962. 75.- Taylor, 1935. 76.- Taylor, 1941a. 77.- Taylor, 1941b. 78.- Taylor, 1972. 79.Torruco et al., 1993. 80.- Villalobos, 1971. 